\title{
Combination of optimal control approaches for aircraft conflict avoidance via velocity regulation
}

\author{
Sonia Cafieri ${ }^{1}$, Loïc Cellier ${ }^{2}$, Frédéric Messine ${ }^{3}$, and Riadh Omheni ${ }^{4}$ \\ ${ }^{1}$ Université de Toulouse, ENAC, Toulouse, France * \\ ${ }^{2}$ Animath, IHP, Paris, France ${ }^{\dagger}$ \\ ${ }^{3}$ Université de Toulouse, LAPLACE, ENSEEIHT-INPT, \\ Toulouse, France ${ }^{\ddagger}$ \\ ${ }^{4}$ SAS Institute, Paris, France $\S$
}

\begin{abstract}
This paper deals with optimal control applied to one of the most crucial and challenging problems in Air Traffic Management, that of aircraft conflict avoidance. We propose an optimal control model where aircraft separation is achieved by changing the speeds of aircraft, and the integral over a time window of their squared accelerations is minimized. Pairwise aircraft separation constraints constitute the main difficulties to be handled. We propose an original decomposition of the problem into three zones, in such a way that in two of them no conflict occurs. Then, using the Pontryagin maximum principle, two new formulations of the original optimal control problem are proposed and solved via direct shooting methods. Thanks to our decomposition, these numerical methods are applied on subproblems having reduced size with respect to the original one, thus improving the efficiency of the solution process. Thirty problem instances are numerically solved, showing the effectiveness of the proposed approaches.
\end{abstract}

*email: sonia.cafieri@enac.fr

†email: lcgo87@gmail.com

† email: frederic.messine@n7.fr

§email: riadh.omheni@sas.com 
Keywords Air traffic management; conflict avoidance; velocity change; optimal control; Pontryagin maximum principle; direct shooting method.

\section{Introduction}

The problem of aircraft conflict avoidance during en-route flights constitutes one of the most crucial and challenging problems in Air Traffic Management (ATM) and Control. It consists in ensuring that aircraft during their flight follow trajectories that respect minimal pairwise distances of separation, horizontally and vertically. When a loss of separation occurs, aircraft are said to be in conflict, and suitable separation maneuvers have to be issued to separate them. This is currently done by air traffic controllers, that continuously monitor some portions of the airspace, and, if a potential conflict is detected, give instruction to separate aircraft. This is crucial to ensure flight safety, and is more and more challenging due to the air traffic growth on the world scale. Increasing automation is therefore needed in ATM, that in turns requires the development of suitable mathematical models and efficient and reliable algorithms.

As one usually aims at solving the problem minimizing the deviation with respect to the original aircraft configuration and flight plans, aircraft conflict avoidance can naturally be modelled as an optimization or an optimal control problem. In this paper, we propose mathematical models and resolution methods based on optimal control.

Standard separation norms for commercial en-route aircraft are 5NM horizontally and $1000 \mathrm{ft}$ vertically $(1 \mathrm{NM}$ (nautical mile) $=1852 \mathrm{~m} ; 1 \mathrm{ft}$ (feet) $=0.3048 \mathrm{~m}$ ). This defines a cylindric protection volume centered on every aircraft, which is then separated from the others if there is no other aircraft entering its protection volume. When aircraft are conflicting, they can be separated deviating for example their heading angle, their altitude or their speed. The first kind of maneuver is currently the most used in practice by air traffic controllers. Aircraft speed variations, on the other hand, are considered very promising, especially in the context of the so-called subliminal speed control [6], consisting in modifying the aircraft speeds within a very small range around their original speeds, without informing air traffic controllers. A review of conflict detection and resolution approaches up to the year 2000 is provided in [19]. A more recent discussion about modelling issues is provided in [8].

Local continuous optimization has been proposed in [21]. Mixed-integer optimization approaches date back to 2002, with linear models proposed in [20, 23]; more recently, nonlinear models are attracting a growing attention, see, e.g., $[1,2,9,10]$. 
Optimal control arises as a natural framework for the problem at hand, as well as for the close problem of trajectory planning, as it allows one to naturally model aircraft dynamics. Clements [13] proposes an optimal control for a minimumtime flight trajectory to a specified fly-to point. The same author in [14] presents a formulation where the control variable constraint is the maximum acceptable aircraft turn rate and the state variable constraint is defined in terms of the radius of the protected zone of conflicting aircraft. Bicchi and Pallottino [3] propose an optimal control model where aircraft velocities are considered constant and again the time for aircraft to go through given trajectory waypoints is minimized. The minimum-time aircraft trajectory problem is also studied in [24], where the optimal controls are found by either maximizing or minimizing a modified Hamiltonian.

In [16], turn and speed changes are considered as controls and some numerical results are obtained, that however show the limitation of the proposed approach in terms of numerical solutions.

Optimal control models in the considered framework are indeed rather difficult to solve. Shooting methods are usually applied (see [5] for a recent survey). Direct shooting methods, based on time discretization, discretize both control and states variables using numerical integrators (of, e.g., Euler or Runge-Kutta type) to approximate the dynamic system. This leads to solve a finite dimensional nonlinear programming (NLP) problem. These methods yield possibly large-scale problems, and in addition suffer of approximation errors due to the time discretization. In contrast, methods based on the Pontryagin Maximum Principle (PMP) [22], map the variables at the initial time to their corresponding values at the final time, that leads to a finite dimensional problem with matching end-point conditions. In this case, there is no time-discretization and a high solution accuracy can be achieved. Nevertheless, these methods suffer of lack of a priori informations about the solution structure, and the initialization of the adjoint (costate) variables is in general difficult. To benefit of the advantages of the two classes of methods, a hybrid approach has been suggested (see, e.g. [7]), where the direct and indirect multiple shooting are combined in such a way that the numerical approximation computed by the direct method is used to initialize the adjoint variables in the indirect shooting method.

In this paper, we propose to use the direct shooting method on new formulations based on the PMP through an original decomposition of the addressed problem. We observe that in aircraft conflict avoidance, we can identify a subproblem where aircraft are potentially conflicting, and so inequality constraints on state variables for pairwise separation have to be applied, and two other subproblems where this kind of constraints do not need to be verified, because aircraft are not yet in the conflict zone or have already passed such a zone (and so, they are separated by construction). 
We propose an optimal control model for aircraft conflict avoidance based on speed regulation, where aircraft accelerations are the commands and where the cost function is the integral on the time window of the squared accelerations. Then, we propose a decomposition of the overall problem in subproblems, that we call prezone, zone, and postzone. Using the PMP on the prezone and on the postzone, we derive analytical solutions. This leads to two new formulations of the original optimal control problem, where the number of variables and constraints in the NLPs obtained by applying direct shooting methods is strongly reduced. We show that these two original resolution strategies allow us to obtain very promising results on problem instances containing up to 13 aircraft.

This paper is organized as follows. In Section 2, we present an optimal control model based on speed changes for aircraft conflict avoidance. In Section 3, we propose our original decomposition of the addressed problem. The main contribution of this paper is presented in Sections 4, 5 and 6, where we present the formulations of the three subproblems, their properties in terms of existence of solutions, and their numerical solution via the application of the PMP or the direct shooting method. In Section 7, we present numerical results validating the proposed approach on a set of 30 problem instances. Section 8 concludes the paper.

\section{Optimal control model through accelera- tion command}

In this section, we present an optimal control model to achieve aircraft separation by only modifying their speeds. This model uses the accelerations of the aircraft as commands of the dynamical system, keeping the trajectories unchanged in terms of headings.

Let us denote $x_{i}$ and $v_{i}$ the state variables representing respectively the position and the velocity of aircraft $i \in I:=\{1, \ldots, \mathrm{n}\}$, where $n$ is the number of aircraft. The acceleration $u_{i}$, for each aircraft $i$, is the command of the system. Aircraft are considered during their en-route cruise phase and expected to be at the same altitude (they are assigned the same flight level), thus we deal with a planar configuration. The velocity $v_{i}$ and so the acceleration $u_{i}$ are with values in $\mathbb{R}$, and the position $x_{i}$ is with values in $\mathbb{R}^{2}$ with $\dot{x}_{i}(t)=v_{i}(t) d_{i}$ where $d_{i} \in \mathbb{R}^{2}$ is fixed. For each aircraft $i$, the velocity $v_{i}$ and the acceleration $u_{i}$ are bounded; i.e., belonging to the interval $\left[\underline{v_{i}}, \overline{v_{i}}\right]$ and $\left[\underline{u_{i}}, \overline{u_{i}}\right]$ respectively.

We consider a tactical en-route phase of flights, that means that an air sector is monitored during a relatively short time window $\left[t_{0}, t_{f}\right]$, and aircraft separation maneuvers are issued in order to have aircraft separated over all this time window. The air sector and the time window can be easily considered in a bijection, i.e., the 
air sector is defined by the positions $x_{i}\left(t_{0}\right)$ and $x_{j}\left(t_{f}\right)$ of all aircraft $i$ and $j$. Thus, the initial and the final times $\left(t_{0}\right.$ and $\left.t_{f}\right)$ are fixed and identical for all aircraft. Let $D$ be the minimum required horizontal separation distance between each pair of aircraft and $d_{i}$ the direction (heading) of the $i^{\text {th }}$ aircraft.

The position $x(t)$ of an aircraft at time $t$ in a planar configuration is to be considered in cartesian coordinates $x(t):=\left(x^{X}(t), x^{Y}(t)\right)^{T}, \forall t \in\left[t_{0}, t_{f}\right]$. Note that the controls $u_{i}$ are searched in a set of square integrable functions over $\left[t_{0}, t_{f}\right]$. Moreover, bound constraints on the controls are directly added in the following problems.

The mathematical model is the following:

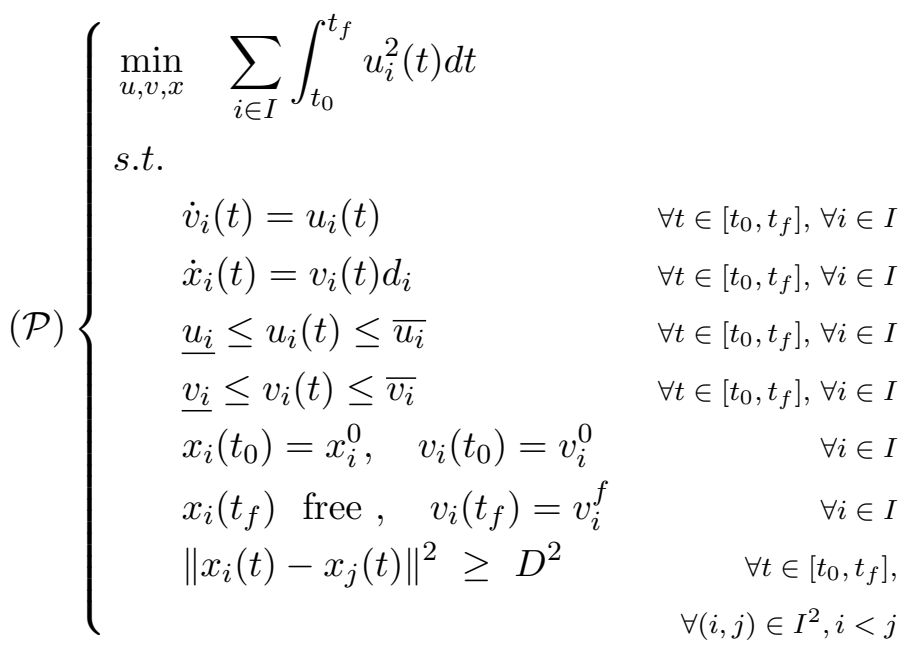

We choose to minimize a quadratic energy cost function depending on speed variations. This criterion takes into account the speed changes of all aircraft by acceleration or deceleration. Note that problem $(\mathcal{P})$ is twice continuously differentiable, with a convex criterion (strictly convex if only $u$ is considered as variable). The state equations, depending on the state variables $v$ and $x$, are all linear. The main difficulties in the solution of $(\mathcal{P})$ are related to the tight bound constraints on the state variables $v_{i}($.$) (specially in the case of subliminal velocity control), and$ to the concave inequality constraints expressing the pairwise aircraft separation.

To solve an optimal control problem, one can derive necessary optimality conditions through the PMP. The solution can then be obtained numerically by applying an indirect shooting technique. However, problem $(\mathcal{P})$ owns constraints involving state variables that makes it difficult to apply such an indirect method. As an alternative to solve problem $(\mathcal{P})$, one can apply direct shooting methods. They are based on the discretization of the time window, yielding a large-scale nonlinear (continuous) optimization problem (NLP), that can be solved by standard local optimization solvers. Note that discretizing problem $(\mathcal{P})$, the number of control 
variables amounts to $n \times p$, where $n$ is the number of aircraft and $p$ is the number of discretization points, and the number of separation constraints amounts to $\frac{n(n-1) p}{2}$ respectively. As an example, let us consider a conflict avoidance problem with only three aircraft, flying during $1 \mathrm{~h}$ discretized by using steps of $15^{\prime \prime}$. This yields a nonlinear problem with $720(n=3$ and $p=240)$ control variables, 720 separation inequality constraints (plus 1440 inequality constraints on the velocity bounds).

In the following, we show that problem $(\mathcal{P})$ can be decomposed into three subproblems in such a way that the PMP can be relatively easily applied to two of them. This implies that the direct shooting method is applied to the remaining subproblem. In this way, the size of the NLP to be solved is drastically reduced, improving the efficiency of the solution process. In the next sections, we present our decomposition method of problem $(\mathcal{P})$ into three parts: prezone, zone and postzone, such that no conflict can occur in the prezone and in the postzone, and we propose a solution approach for each of these subproblems.

\section{Decomposing the problem into zones}

Given the aircraft predicted planar (straight-line) trajectories, one can check intersections of these trajectories so as to identify spatial regions where the separation constraints must be actually checked. The corresponding time window where the separation constraints have to be checked can then be identified as well. This is the basis of the original strategy of resolution of the problem at hand, proposed in this paper. In the following, we discuss the decomposition of the problem.

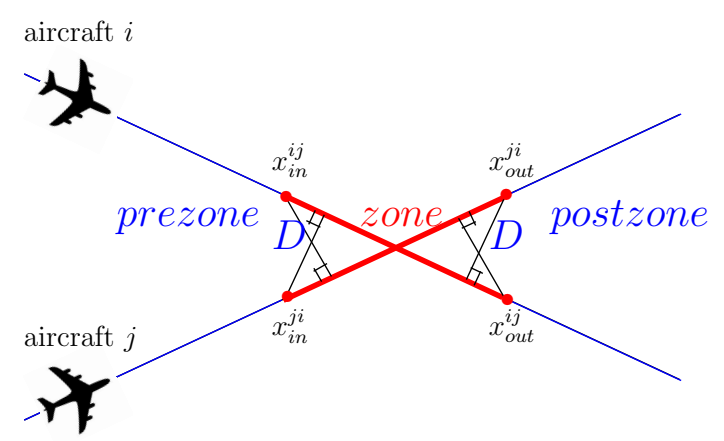

Figure 1: Spatial decomposition for two aircraft.

Let $S_{i}$ define the line segment corresponding to all the positions $x_{i}(t)$ of aircraft $i$, for all time $t$ in the time window $\left[t_{0}, t_{f}\right]$. Two aircraft $i$ and $j$ are in conflict when some points on $S_{i}$ have a distance less than or equal to $D$ to another point on $S_{j}$, and reciprocally (see Fig. 1). We can thus easily identify a planar region where $i$ 
and $j$ are in conflict. For each pair of aircraft, we will denote this planar region by zone. The zone is characterized by the fact that no conflict occurs outside it, while inside it conflicts may occur, and so separation constraints have to be imposed. We define prezone the region preceding the zone, and respectively postzone the remaining region. This is illustrated in Fig. 1.

In the remaining of this section, we present the way a zone is identified in practice. Let us consider aircraft $i$ and $j$. We first have to determine their entrance and exit points $x_{i n}^{i j}$ and $x_{o u t}^{i j}$, if they exist. This means that we have to check if $S_{i}$ and $S_{j}$ own points at a distance less than or equal to $D$. Let us denote by $\left(D_{i}\right): a_{i} x+b_{i} y+c_{i}=0$ the equation of the line supporting $S_{i}$; this defines the equation of the trajectory of aircraft $i$ and so $a_{i}=d_{i}^{Y}, b_{i}=-d_{i}^{X}$, and $c_{i}=$ $-a_{i} x_{i}^{X}\left(t_{0}\right)-b_{i} x_{i}^{Y}\left(t_{0}\right)$. For $i$ and $j$ following trajectories $\left(D_{i}\right)$ and $\left(D_{j}\right)$ respectively, to determine the points $x_{i n}^{i j}$ and $x_{\text {out }}^{i j}$ (if they exist), we use the fact that $x_{i n}^{i j}$ and $x_{\text {out }}^{i j}$ must belong to $\left(D_{i}\right)$, for aircraft $i$, and have distance from $\left(D_{j}\right)$ equal to $D$ (reciprocally for aircraft $j$ ). This yields the following two equations:

$$
\begin{aligned}
& a_{i} x+b_{i} y+c_{i}=0 \\
& \frac{\left|a_{j} x+b_{j} y+c_{j}\right|}{\sqrt{a_{j}^{2}+b_{j}^{2}}}=D
\end{aligned}
$$

that give two distinct but similar linear systems:

$$
\left\{\begin{array} { l } 
{ a _ { i } x + b _ { i } y = - c _ { i } } \\
{ a _ { j } x + b _ { j } y = D \sqrt { a _ { j } ^ { 2 } + b _ { j } ^ { 2 } } - c _ { j } }
\end{array} \quad \left\{\begin{array}{l}
a_{i} x+b_{i} y=-c_{i} \\
a_{j} x+b_{j} y=-D \sqrt{a_{j}^{2}+b_{j}^{2}}-c_{j}
\end{array}\right.\right.
$$

Denoting by $\left(K_{i}, K_{j}\right)^{T}$ the second right hand side of the first system (for the second one, we just have to change a sign in $K_{j}$ ), we obtain:

$$
x=\frac{b_{j} K_{i}-b_{i} K_{j}}{a_{i} b_{j}-a_{j} b_{i}} \text { and } y=\frac{a_{j} K_{i}-a_{i} K_{j}}{a_{j} b_{i}-a_{i} b_{j}} .
$$

Depending on the values of $K_{j}$, these yield two distinct points which are $x_{i n}^{i j}$ and $x_{\text {out }}^{i j}$. We just have to check which point is in and which one is out, and also if they exist and belong to $S_{i}$. Note that this computation, while being easy, has to be done twice for each pair of aircraft. This therefore yields $n \times(n-1)$ computations.

Once entry and exit points $x_{i n}^{i j}$ and $x_{o u t}^{i j}$ are known, we can determine the time $z o n e$ for an aircraft $i$, i.e., the time window corresponding to the zone. We denote it by $\left[t_{\text {in }}^{i}, t_{\text {out }}^{i}\right]$ (see Fig. 2). We have to solve the following equations: $\dot{x}_{i}(t)=\bar{v}_{i} d_{i}$ and $\dot{x}_{i}(t)=\underline{v_{i}} d_{i}$ to obtain $t_{\text {in }}^{i}$ and $t_{\text {out }}^{i}$ respectively. Considering $\dot{x}_{i}(t)=\bar{v}_{i} d_{i}$, one 
has $x_{i}(t)=\overline{v_{i}} d_{i} t+K_{1}$, where $K_{1}:=x_{i}\left(t_{0}\right)-\overline{v_{i}} d_{i} t_{0}$ and thus

$$
t_{i n}^{i j}:=\left\{\begin{array}{l}
\frac{\left(x_{i n}^{i j}\right)^{X}-K_{1}}{\overline{v_{i}} d_{i}^{X}}, \text { if } d_{i}^{X} \neq 0 \\
\frac{\left(x_{i n}^{i j}\right)^{Y}-K_{1}}{\overline{v_{i}} d_{i}^{Y}}, \text { else. }
\end{array}\right.
$$

Considering now the second equation, we obtain similarly:

$$
t_{\text {out }}^{i j}:=\left\{\begin{array}{l}
\frac{\left(x_{\text {out }}^{i j}\right)^{X}-K_{2}}{\underline{v_{i}} d_{i}^{X}}, \text { if } d_{i}^{X} \neq 0 \\
\frac{\left(x_{\text {out }}^{i j}\right)^{Y}-K_{2}}{\underline{v_{i}} d_{i}^{Y}}, \text { else. }
\end{array}\right.
$$

where $K_{2}:=x_{i}\left(t_{0}\right)-\underline{v_{i}} d_{i} t_{0}$.
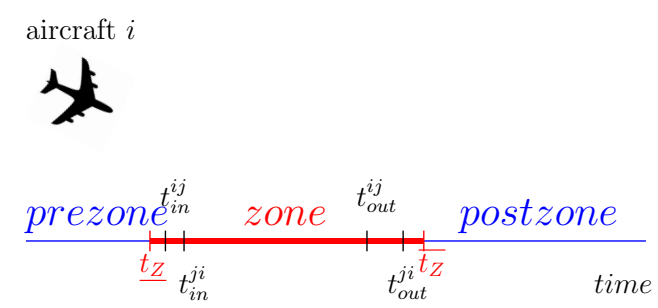

aircraft $j$

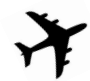

Figure 2: Decomposition in time zones for all aircraft. Time $t_{\text {in }}$ and $t_{\text {out }}$ are shown for aircraft $i$ and $j$.

Remark that for $i$ and $j$, if $t_{i n}^{i j} \neq t_{i n}^{j i}$, we take the maximum value, and similarly for $t_{\text {out }}^{i j} \neq t_{\text {out }}^{j i}$. Finally, we can define the general time zone $\left[\underline{t_{Z}}, \overline{t_{Z}}\right]$ as:

$$
\underline{t_{Z}}:=\min _{i \in I} \min _{j \in I, j>i} \max \left\{t_{i n}^{i j}, t_{i n}^{j i}\right\} \quad \text { and } \quad \overline{t_{Z}}:=\max _{i \in I} \max _{j \in I, j>i} \min \left\{t_{\text {out }}^{i j}, t_{\text {out }}^{j i}\right\}
$$

that so requires a computational complexity of $O\left(n^{2}\right)$. The general time zone, illustrated in Fig. 2, corresponds to a unique time window for all the $n$ aircraft and it is the only window where aircraft separation constraints have to be satisfied. 


\section{Applying the PMP on the postzone}

In this section, we focus on the postzone to derive necessary optimality conditions using the PMP.

From a temporal point of view, the postzone corresponds to $\left[t_{Z}, t_{f}\right]$, i.e., from the end of the zone until the final time. As conflicts occur only in the zone, starting from $\overline{t_{Z}}$, there is no more conflict between the aircraft. Thus, the aircraft separation constraints can be discarded yielding the following problem:

$$
\left(\mathcal{P}_{\text {postzone }}\right)\left\{\begin{array}{lr}
\min _{u, v, x} \sum_{i \in I} \int_{\overline{t_{Z}}}^{t_{f}} u_{i}^{2}(t) d t & \\
\text { s.t. } & \forall t \in\left[\overline{t_{Z}}, t_{f}\right], \forall i \in I \\
\dot{v}_{i}(t)=u_{i}(t) & \forall t \in\left[t_{\bar{t}}, t_{f}\right], \forall i \in I \\
\dot{x}_{i}(t)=v_{i}(t) d_{i} & \forall t \in\left[\overline{t_{Z}}, t_{f}\right], \forall i \in I \\
\underline{u_{i}} \leq u_{i}(t) \leq \overline{u_{i}} & \forall t \in\left[t_{Z}, t_{f}\right], \forall i \in I \\
\underline{v_{i}} \leq v_{i}(t) \leq \overline{v_{i}} & \forall i \in I \\
x_{i}\left(\overline{t_{Z}}\right)=x_{i}^{\overline{t_{Z}}}, \quad v_{i}\left(\overline{t_{Z}}\right)=v_{i}^{\overline{t_{Z}}} & \forall i \in I \\
x_{i}\left(t_{f}\right) \text { free }, \quad v_{i}\left(t_{f}\right)=v_{i}^{f} &
\end{array}\right.
$$

Remark 1 The fixed values of the velocity at times $\overline{t_{Z}}$ and $t_{f}$, respectively $v_{i}^{\overline{t_{Z}}}$ and $v_{i}^{f}$, have to be in the interval $\left[\underline{v_{i}}, \overline{v_{i}}\right]$, otherwise it would not be any point satisfying the constraints of problem $(\mathcal{P}$ postzone $)$. In the following, we suppose that the values of $v_{i}^{\overline{t_{Z}}}$ and $v_{i}^{f}$ are in the interval.

The following two propositions provide some properties about the solution of problem $(\mathcal{P}$ postzone $)$.

Proposition 1 (Conditions on the existence of a realizable solution) A realizable solution of problem $\left(\mathcal{P}_{\text {postzone }}\right)$ exists if and only if

$$
\underline{u_{i}} \leq \frac{v_{i}^{f}-v_{i}^{\overline{t_{Z}}}}{t_{f}-\overline{t_{Z}}} \leq \overline{u_{i}}, \quad \forall i \in I .
$$

Proof 1 Let $\tilde{u}$ be a realizable control, that implies $\underline{u_{i}} \leq \tilde{u}_{i}(t) \leq \overline{u_{i}} \quad \forall t \in\left[\overline{t_{Z}}, t_{f}\right], \forall i \in$ $I$.

By using the monotonicity property of the integral, one has:

$$
\int_{\overline{t_{Z}}}^{t_{f}} \underline{u_{i}} \leq \int_{\overline{t_{Z}}}^{t_{f}} \tilde{u}_{i}(t) d t \leq \int_{\overline{t_{Z}}}^{t_{f}} \overline{u_{i}} \quad \forall i \in I .
$$


Hence, denoting by $\tilde{v}_{i}$ the velocity corresponding to the acceleration $\tilde{u}_{i}$, one obtains

$$
\left(t_{f}-\overline{t_{Z}}\right) \times \underline{u_{i}} \leq \tilde{v}_{i}\left(t_{f}\right)-\tilde{v}_{i}\left(\overline{t_{Z}}\right) \leq\left(t_{f}-\overline{t_{Z}}\right) \times \overline{u_{i}} \quad \forall t \in\left[\overline{t_{Z}}, t_{f}\right], \quad \forall i \in I .
$$

The result follows from $\tilde{v}_{i}\left(\overline{t_{Z}}\right)=v_{i}^{\overline{t_{Z}}}$ and $\tilde{v}_{i}\left(t_{f}\right)=v_{i}^{f}$.

Reciprocally, suppose that

$$
\underline{u_{i}} \leq \frac{v_{i}^{f}-v_{i}^{\overline{t_{Z}}}}{t_{f}-\overline{t_{Z}}} \leq \overline{u_{i}}, \quad \forall i \in I .
$$

By considering a constant control $\tilde{u}_{i}(t):=\frac{v_{i}^{f}-v_{i}^{\overline{t_{Z}}}}{t_{f}-\overline{t_{Z}}}, \forall t \in\left[\overline{t_{Z}}, t_{f}\right], \forall i \in I$, we obtain the corresponding velocity:

$\tilde{v}_{i}(t)=\int_{\overline{t_{Z}}}^{t} \tilde{u}_{i}(\tau) d \tau+\tilde{v}_{i}\left(\overline{t_{Z}}\right)=\int_{\overline{t_{Z}}}^{t} \frac{v_{i}^{f}-v_{i}^{\overline{t_{Z}}}}{t_{f}-\overline{t_{Z}}} d \tau+\tilde{v}_{i}\left(\overline{t_{Z}}\right)=\left(t-\overline{t_{Z}}\right) \times \frac{v_{i}^{f}-v_{i}^{\overline{t_{Z}}}}{t_{f}-\overline{t_{Z}}}+\tilde{v}_{i}\left(\overline{t_{Z}}\right)$.

Thus, $\quad \tilde{v}_{i}\left(t_{f}\right)=\left(t_{f}-\overline{t_{Z}}\right) \times \frac{v_{i}^{f}-v_{i}^{\overline{t_{Z}}}}{t_{f}-\bar{t}_{Z}}+\tilde{v}_{i}\left(\overline{t_{Z}}\right)=v_{i}^{f}-v_{i}^{\overline{t_{Z}}}+\tilde{v}_{i}\left(\overline{t_{Z}}\right)$.

Hence, setting $\tilde{v}_{i}\left(\overline{t_{Z}}\right):=v_{i}^{\overline{t_{Z}}}$, one has $\tilde{v}_{i}\left(t_{f}\right)=v_{i}^{f}$. Moreover, as $\tilde{v}_{i}$ is affine with respect to $t$, and as $v_{i}^{\overline{t_{Z}}}$ and $v_{i}^{f}$ are in $\left[\underline{v_{i}}, \overline{v_{i}}\right]$, one has that $\tilde{v}_{i}(t) \in\left[\underline{v_{i}}, \overline{v_{i}}\right]$ for all $t \in\left[t_{Z}, t_{f}\right]$.

Therefore, this solution $(\tilde{u}, \tilde{v})$ is realizable for problem $(\mathcal{P}$ postzone $)$. Note that there is no constraint on the final position and so, $\tilde{x}$ is just defined from $\tilde{v}$ by fixing $\tilde{x}\left(\overline{t_{Z}}\right)=x^{\overline{t_{Z}}}$.

Proposition 2 (Solution of problem (P $\left.\mathcal{P}_{\text {postzone }}\right)$ ) The unique solution of problem $\left(\mathcal{P}_{\text {postzone }}\right)$, if a realizable solution exists, is:

$$
u_{i}^{*}(t):=\frac{v_{i}^{f}-v_{i}^{\overline{t_{Z}}}}{t_{f}-\overline{t_{Z}}}, \quad \forall i \in I .
$$

This optimal control provides the following optimal state variables:

$$
\begin{aligned}
v_{i}^{*}(t) & :=\frac{v_{i}^{f}-v_{i}^{\overline{t_{Z}}}}{t_{f}-\overline{t_{Z}}} t+\frac{v_{i}^{\overline{t_{Z}}} \times t_{f}-v_{i}^{f} \times \overline{t_{Z}}}{t_{f}-\overline{t_{Z}}} \\
\text { and } & \\
x_{i}^{*}(t) & :=d_{i} \times\left(\frac{v_{i}^{f}-v_{i}^{\overline{t_{Z}}}}{2\left(t_{f}-\overline{t_{Z}}\right)} t^{2}+\frac{v_{i}^{\bar{t}_{Z}} \times t_{f}-v_{i}^{f} \times \overline{t_{Z}}}{t_{f}-\overline{t_{Z}}} t\right)+X^{0}
\end{aligned}
$$


where $X^{0}=x^{\overline{t_{Z}}}-d_{i} \times\left(\frac{v_{i}^{f}-v_{i}^{\overline{t_{Z}}}}{2\left(t_{f}-\overline{t_{Z}}\right)} \bar{t}^{2}+\frac{v_{i}^{\overline{t_{Z}}} \times t_{f}-v_{i}^{f} \times \overline{t_{Z}}}{t_{f}-\overline{t_{Z}}} \overline{t_{Z}}\right)$, and the following optimal cost

$$
J^{*}:=J\left(u^{*}\right)=\sum_{i \in I} \frac{\left(v_{i}^{f}-v_{i}^{\overline{t_{Z}}}\right)^{2}}{t_{f}-\overline{t_{Z}}} .
$$

Proof 2 Let us first relax problem ( $\mathcal{P}_{\text {postzone }}$ ) by discarding bound constraints on $u$ and $v: \quad \underline{u_{i}} \leq u_{i}(t) \leq \overline{u_{i}}$ and $\underline{v_{i}} \leq v_{i}(t) \leq \overline{v_{i}}, \quad \forall t \in\left[\overline{t_{Z}}, t_{f}\right] \forall i \in I$.

Note that problem $(\mathcal{P}$ postzone $)$ corresponds to the sum of $n$ independent subproblems, one for each $i \in I$, as follows:

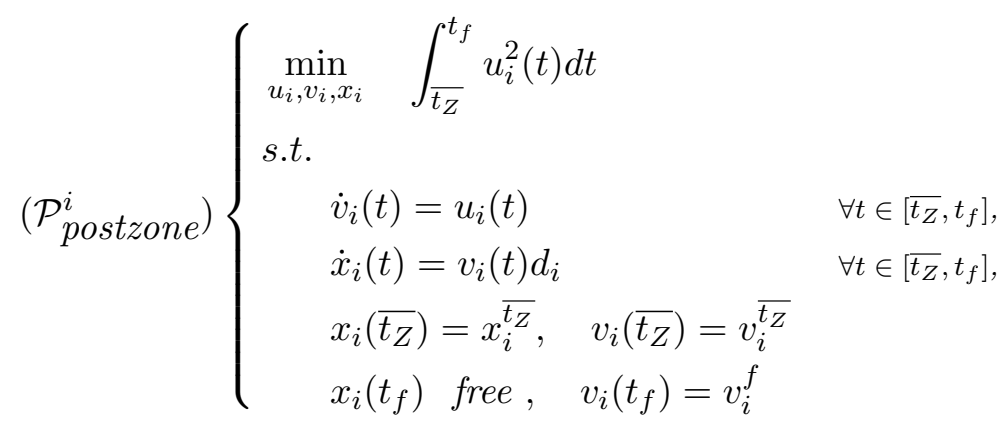

The Hamiltonian of problem $\left(\mathcal{P}_{\text {postzone }}^{i}\right)$ is:

$$
\begin{aligned}
& H_{i}\left(x_{i}^{X}(t), x_{i}^{Y}(t), v_{i}(t), z_{1, i}(t), z_{2, i}(t), z_{3, i}(t), z_{0, i}, u_{i}(t)\right) \\
& =z_{1, i}(t) v_{i}(t) d_{i}^{X}+z_{2, i}(t) v_{i}(t) d_{i}^{Y}+z_{3, i}(t) u_{i}(t)+z_{0, i} u_{i}^{2}(t),
\end{aligned}
$$

where the real scalar $z_{0, i}$ is associated to the cost function and $z_{1, i}(t), z_{2, i}(t), z_{3, i}(t)$ are the costate variables; $z_{1, i}(t), z_{2, i}(t), z_{3, i}(t)$ are associated to $x_{i}^{X}(t), x_{i}^{Y}(t)$ and $v_{i}(t)$ respectively. 
The associated system of differential equations, for all $t \in\left[t_{Z}, t_{f}\right]$, is:

$$
\begin{aligned}
& \dot{x}_{i}^{X}(t)=\frac{\partial H_{i}(.)}{\partial z_{1, i}}=v_{i}(t) d_{i}^{X}, \\
& \dot{x}_{i}^{Y}(t)=\frac{\partial H_{i}(.)}{\partial z_{2, i}}=v_{i}(t) d_{i}^{Y}, \\
& \dot{v}_{i}(t)=\frac{\partial H_{i}(.)}{\partial z_{3, i}}=u_{i}(t), \\
& \dot{z}_{1, i}(t)=-\frac{\partial H_{i}(.)}{\partial x_{i}^{X}}=0, \\
& \dot{z}_{2, i}(t)=-\frac{\partial H_{i}(.)}{\partial x_{i}^{Y}}=0, \\
& \dot{z}_{3, i}(t)=-\frac{\partial H_{i}(.)}{\partial v_{i}}=-z_{1, i}(t) d_{i}^{X}-z_{2, i}(t) d_{i}^{Y} .
\end{aligned}
$$

Maximizing the Hamiltonian with respect to $u_{i}$, and then fixing the value $z_{0, i}$ to -1 without loss of generality (just the normal extremals are studied here), we obtain:

$$
u_{i}^{*}=\underset{u_{i}}{\operatorname{argmax}} H_{i}(.)=\frac{z_{3, i}(t)}{2}
$$

This result is obtained by deriving $H_{i}$ with respect to $u_{i}\left(\frac{\partial H_{i}}{\partial u_{i}}=z_{3, i}-2 u_{i}\right)$, and by solving $\frac{\partial H_{i}}{\partial u_{i}}=0$, thus providing the necessary optimality conditions at the first order. By deriving two times with respect to $u_{i}$, we obtain $\frac{\partial^{2} H_{i}}{\partial u_{i}^{2}}=-2<0$, which proves that $u_{i}^{*}$ is indeed the unique maximum of $H_{i}$.

By replacing $u_{i}(t)$ in the above differential equations by $\frac{z_{3, i}(t)}{2}$, this yields a system with 6 equations, 6 unknowns, 3 initial conditions and just 1 final condition. The transversality conditions provide 2 new final conditions on the costate variables: $z_{1, i}\left(t_{f}\right)=0$ and $z_{2, i}\left(t_{f}\right)=0$. Considering the two differential equations $\dot{z}_{1, i}(t)=0$ and $\dot{z}_{2, i}(t)=0$, this implies that $z_{1, i}(t)=0$ and $z_{1, i}(t)=0$, for all $t \in$ $\left[t_{Z}, t_{f}\right]$. Therefore, the last differential equation $\dot{z}_{3, i}(t)=-z_{1, i}(t) d_{i}^{X}-z_{2, i}(t) d_{i}^{Y}=0$, and then $z_{3, i}$ is constant for all $t \in\left[\overline{t_{Z}}, t_{f}\right]$. By denoting $A$ this constant, one has $u_{i}^{*}(t)=\frac{A}{2}$ and hence (considering the third differential equation of our system), $v_{i}^{*}(t)=\frac{A}{2} t+B$, where $B$ is another real constant. By using the initial and $f_{-}$nal conditions on $v_{i}$, one obtains the values of the two constants $A$ and $B$; i.e., $v_{i}^{*}\left(\overline{t_{Z}}\right)=\frac{A}{2} \overline{t_{Z}}+B=v_{i}^{\overline{t_{Z}}}$ and $v_{i}^{*}\left(t_{f}\right)=\frac{A}{2} t_{f}+B=v_{i}^{f}$, hence $A=2 \frac{v_{i}^{f}-v_{i}^{\overline{t_{Z}}}}{t_{f}-\bar{t}_{Z}}$ and $B=\frac{t_{f} \times v_{i}^{\bar{t}}-\overline{t_{Z}} \times v_{i}^{f}}{t_{f}-\overline{t_{Z}}}$. Substituting the values of these two constants, we obtain the expression of the optimal solution $u^{*}, v^{*}, x^{*}$ and $J^{*}$. 
Let us now discuss about the relaxed bound constraints on $u$ and $v$.

First consider the bound constraints on the velocity $v: \underline{v_{i}} \leq v_{i}(t) \leq \overline{v_{i}}$. From the values of $A$ and $B$ obtained by solving the relaxed problem, one has that $v_{i}^{*}(t)=$ $\frac{A}{2} t+B$ and then $v_{i}^{*}(t)$ is affine. Let us suppose that $v_{i}^{f} \leq v_{i}^{\overline{t_{Z}}}$, then one has $A \leq 0$ and then $v_{i}^{*}(t) \leq v_{i}^{*}\left(\overline{t_{Z}}\right)=v_{i}^{\overline{t_{Z}}}$ for all $t \in\left[\overline{t_{Z}}, t_{f}\right]$. Moreover, as $A \leq 0$, one has $v_{i}^{*}(t) \geq v_{i}^{*}\left(t_{f}\right)=v_{i}^{f}$ for all $t \in\left[\overline{t_{Z}}, t_{f}\right]$. Hence, $\underline{v}_{i} \leq v_{i}^{f} \leq v_{i}^{*}(t) \leq v_{i}^{\overline{t_{Z}}} \leq \overline{v_{i}}$, for all $t \in\left[t_{Z}, t_{f}\right]$. Let us now suppose that $v_{i}^{f} \geq v_{i}^{\overline{t_{Z}}}$, then one has $A \geq 0$ and then $v_{i}^{*}(t) \geq v_{i}^{*}\left(\overline{t_{Z}}\right)=v_{i}^{\overline{t_{Z}}}$ for all $t \in\left[\overline{t_{Z}}, t_{f}\right]$. Moreover, as $A \geq 0$, one has $v_{i}^{*}(t) \leq v_{i}^{*}\left(t_{f}\right)=v_{i}^{f}$ for all $t \in\left[\overline{t_{Z}}, t_{f}\right]$. Thus, one has $\underline{v_{i}} \leq v_{i}^{\overline{t_{Z}}} \leq v_{i}^{*}(t) \leq v_{i}^{f} \leq \overline{v_{i}}$, for all $t \in\left[t_{Z}, t_{f}\right]$. In conclusion, if the optimal control $u^{*}$ exists then the bound constraints on the velocity $v$ are satisfied.

Let us now consider the bound constraints on $u: \underline{u_{i}} \leq u_{i}(t) \leq \overline{u_{i}}$ for all $i \in I$ and $t \in\left[\overline{t_{Z}}, t_{f}\right] . \quad$ As $u_{i}^{*}(t)$ is constant and equal to $\frac{\overline{v_{i}^{f}-v_{i}^{\overline{t_{Z}}}}}{t_{f}-\overline{t_{Z}}}$, if $\exists i \in I$ such that $\frac{v_{i}^{f}-v_{i}^{\bar{t}}}{t_{f}-\bar{t}_{Z}} \notin\left[\underline{u_{i}}, \overline{u_{i}}\right]$ from Proposition 1, one can conclude that there is no realizable control for problem $\left(\mathcal{P}\right.$ postzone). In conclusion, if a control $u^{*}$ exists, the bound constraints on $u$ are satisfied.

Therefore, we proved that from the relaxed problem $\left(\mathcal{P}_{\text {postzone }}\right)$, the optimal control $u^{*}$ can be provided using the PMP; the velocity, the position and the final cost can then be derived. Moreover, such an optimal constant solution $u^{*}$ exists if and only if the bound constraints on $u$ are satisfied.

Remark 2 In the addressed application, the bounds on the control are in practice quite large, and so easily satisfied. It follows that in general, we have a solution of problem $(\mathcal{P}$ postzone $)$.

By decomposing the time interval into two parts $\left[t_{0}, \overline{t_{Z}}\right]$ and $\left[t_{Z}, t_{f}\right]$, and using Propositions 1 and 2, one can reformulate problem $(\mathcal{P})$. The following theorem presents this new formulation of problem $(\mathcal{P})$, involving a lower number of variables with respect to the initial one.

Theorem 1 (Reformulation of problem $(\mathcal{P})$ ) A reformulation of problem $(\mathcal{P})$ 
is:

$$
\left(\mathcal{P}_{1}\right)\left\{\begin{array}{lr}
\min _{u, v, x} \sum_{i \in I} \int_{t_{0}}^{\overline{t_{Z}}} u_{i}^{2}(t) d t & +\sum_{i \in I} \frac{\left(v_{i}^{f}-v_{i}\left(\overline{t_{Z}}\right)\right)^{2}}{t_{f}-\overline{t_{Z}}} \\
\text { s.t. } & \forall t \in\left[t_{0}, \overline{t_{Z}}\right], \forall i \in I \\
\dot{v}_{i}(t)=u_{i}(t) & \forall t \in\left[t_{0}, \overline{t_{Z}},, \forall i \in I\right. \\
\dot{x}_{i}(t)=v_{i}(t) d_{i} & \forall t \in\left[t_{0}, \overline{t_{Z}}\right], \forall i \in I \\
\underline{u_{i}} \leq u_{i}(t) \leq \overline{u_{i}} & \forall t \in\left[t_{0}, \overline{t_{Z}},, \forall i \in I\right. \\
\underline{v_{i}} \leq v_{i}(t) \leq \overline{v_{i}} & \forall i \in I \\
\underline{u_{i}} \leq \frac{v_{i}^{f}-v_{i}\left(\overline{t_{Z}}\right)}{t_{f}-\overline{\bar{t}_{Z}} \leq \overline{u_{i}}} & \forall i \in I \\
x_{i}\left(t_{0}\right)=x_{i}^{0}, \quad v_{i}\left(t_{0}\right)=v_{i}^{0} & \forall i \in I \\
x_{i}\left(t_{f}\right) \text { free }, \quad v_{i}\left(t_{f}\right)=v_{i}^{f} & \forall t \in\left[t_{0}, \overline{t_{Z}}\right], \\
\left\|x_{i}(t)-x_{j}(t)\right\|^{2} \geq D^{2} & \forall(i, j) \in I^{2}, i<j
\end{array}\right.
$$

Proof 3 By decomposing the time window $\left[t_{0}, t_{f}\right]$ into two parts $\left[t_{0}, \overline{t_{Z}}\right]$ and $\left[t_{Z}, t_{f}\right]$, problem $(\mathcal{P})$ becomes almost separable. The only link occurs at time $\overline{t_{Z}}$. Indeed, the two subproblems can provide disconnected solutions of $u, v$ and $x$, but only at time $\overline{t_{Z}}$.

The control $u$ can be discontinuous because it is possible for an aircraft to change roughly its acceleration. So u can be discontinuous at time $\overline{t_{Z}}$; indeed, $u$ has just to be integrable over $\left[t_{0}, \overline{t_{Z}}\right]$ and $\left[t_{Z}, t_{f}\right]$. For the velocity $v$ and the position $x$, one must keep the continuity of the solution at time $\overline{t_{Z}}$. Suppose that we solve the first subproblem on $\left[t_{0}, \overline{t_{Z}}\right]$, computing an optimal control $u^{1}$, an optimal velocity $v^{1}$ and position $x^{1}$. Using the values of $v^{1}\left(\overline{t_{Z}}\right)$ and $x^{1}\left(\overline{t_{Z}}\right)$ used as initial conditions of the second subproblem for $t$ in $\left[t_{Z}, t_{f}\right]$, and from Propositions 1 and 2, a solution of the second subproblem, if it exists, can be easily computed. The existence of a solution for the second subproblem provides a new constraint on $v^{1}\left(\overline{t_{Z}}\right): \underline{u_{i}} \leq \frac{v_{i}^{f}-v_{i}^{1}\left(\overline{t_{Z}}\right)}{t_{f}-\overline{t_{Z}}} \leq \overline{u_{i}}$ for all $i \in I$. Note that if this constraint is not satisfied, the second subproblem (for time in $\left[t_{Z}, t_{f}\right]$ ) has no realizable point (see Proposition 1). Therefore this constraint has to be added to problem $\left(\mathcal{P}_{Z 1}\right)$ and the result follows.

Remark 3 The new formulation of Theorem 1 only provides solutions for all $t \in\left[t_{0}, \overline{t_{Z}}\right]$, if such an optimal solution exists. All the remaining solutions, for all time $t$ in $\left[t_{Z}, t_{f}\right]$, have to be derived from Proposition 2. 


\section{Applying the PMP on the prezone}

In this section, we focus on the prezone to derive necessary optimality conditions using the PMP. This provides a new formulation of problem $(\mathcal{P})$.

This section follows the same outline as the previous one dedicated to the study of the postzone, however the case treated here is much more difficult and major differences occur. For the sake of simplification and without loss of generality, we fix $t_{0}=0$ in this section.

From a temporal point of view, the prezone corresponds to $\left[0, \underline{t}_{Z}\right]$, i.e., from the initial time until the beginning of the zone. As conflicts occur only in the zone, from the initial time to the beginning of the zone, $t_{Z}$, there is no conflict between aircraft. Thus, the aircraft separation constraints can be discarded yielding the following problem:

$$
\left(\mathcal{P}_{\text {prezone }}\right)\left\{\begin{array}{lr}
\min _{u, v, x} \sum_{i \in I} \int_{0}^{\underline{t_{Z}}} u_{i}^{2}(t) d t & \\
\text { s.t. } & \forall t \in\left[t_{0}, \underline{t_{Z}}\right], \forall i \in I \\
\dot{v}_{i}(t)=u_{i}(t) & \forall t \in\left[t_{0}, \underline{t_{Z}}\right], \forall i \in I \\
\dot{x}_{i}(t)=v_{i}(t) d_{i} & \forall t \in\left[t_{0}, \underline{t_{Z}}\right], \forall i \in I \\
\underline{u_{i}} \leq u_{i}(t) \leq \overline{u_{i}} & \forall t \in\left[t_{0}, \underline{t_{Z}}\right], \forall i \in I \\
\underline{v_{i}} \leq v_{i}(t) \leq \overline{v_{i}} & \forall i \in I \\
x_{i}(0)=x_{i}^{0}, \quad v_{i}(0)=v_{i}^{0} & \forall i \in I \\
x_{i}\left(\underline{t_{Z}}\right)=x_{i}^{t_{Z}}, \quad v_{i}\left(\underline{t_{Z}}\right)=v_{i}^{\underline{t_{Z}}} &
\end{array}\right.
$$

Remark 4 The only difference between problem $\left(\mathcal{P}_{\text {postzone }}\right)$ and problem $\left(\mathcal{P}_{\text {prezone }}\right)$ is that the final position has now to be fixed: $x_{i}\left(\underline{t_{Z}}\right)=x_{i}^{t_{Z}}$.

Remark 5 The fixed values of the velocity at times 0 and $\underline{t_{Z}}$, respectively $v_{i}^{0}$ and $v_{i}^{t_{Z}}$, have to be in the interval $\left[v_{i}, \overline{v_{i}}\right]$, otherwise it would not exist any point satisfying the constraints of problem $(\mathcal{P}$ prezone).

In the following, we suppose that the values of $v_{i}^{0}$ and $v_{i}^{t_{Z}}$ are in the interval $\left[v_{i}, \overline{v_{i}}\right]$.

In the first part of this section the bound constraints on the velocity are not 
taken into account, then the problem (P prezone) is relaxed providing:

$$
\left(\mathcal{P}_{\text {R_prezone }}\right)\left\{\begin{array}{lr}
\min _{u, v, x} \sum_{i \in I} \int_{0}^{\underline{t_{Z}}} u_{i}^{2}(t) d t & \\
\text { s.t. } & \\
\dot{v}_{i}(t)=u_{i}(t) & \forall t \in\left[t_{0}, \underline{t_{Z}}\right], \forall i \in I \\
\dot{x}_{i}(t)=v_{i}(t) d_{i} & \forall t \in\left[t_{0}, \underline{t_{Z}}\right], \forall i \in I \\
\underline{u_{i} \leq u_{i}(t) \leq \overline{u_{i}}} & \forall t \in\left[t_{0}, \underline{t_{Z}}\right], \forall i \in I \\
x_{i}(0)=x_{i}^{0}, \quad v_{i}(0)=v_{i}^{0} & \forall i \in I \\
x_{i}\left(\underline{t_{Z}}\right)=x_{i}^{t_{Z}}, \quad v_{i}\left(\underline{t_{Z}}\right)=v_{i}^{\underline{t_{Z}}} & \forall i \in I
\end{array}\right.
$$

The way to consider the bound constraints on the velocity will be discussed below to derive a new formulation of problem $(\mathcal{P})$.

The following two propositions provide some properties about the solution of problem $\left(\mathcal{P}_{\mathrm{R}_{-} \text {prezone }}\right)$.

Proposition 3 (A solution of Problem $\left(\mathcal{P}_{\mathbf{R} \_ \text {prezone }}\right)$ ) If

$$
\underline{u_{i}} \leq \frac{6\left(\left(x_{i}^{t_{Z}}\right)^{D}-\left(x_{i}^{0}\right)^{D}\right)}{d_{i}^{D} \underline{t}^{2}}-\frac{2\left(v_{i}^{t_{Z}}+2 v_{i}^{0}\right)}{\underline{t_{Z}}} \leq \overline{u_{i}}
$$

and

$$
\underline{u_{i}} \leq \frac{-6\left(\left(x_{i}^{t_{Z}}\right)^{D}-\left(x_{i}^{0}\right)^{D}\right)}{d_{i}^{D} \underline{t_{Z}}}+\frac{4\left(v_{i}^{t_{Z}}+v_{i}^{0}\right)}{\underline{t_{Z}}} \leq \overline{u_{i}}
$$

Then one has

$$
\begin{aligned}
& u_{i}^{*}(t)=-\left(\frac{12\left(\left(x_{i}^{t_{Z}}\right)^{D}-\left(x_{i}^{0}\right)^{D}\right)}{d_{i}^{D} \underline{t Z}^{3}}-\frac{6\left(v_{i}^{t_{Z}}+v_{i}^{0}\right)}{{\underline{t_{Z}}}^{2}}\right) t+\frac{6\left(\left(x_{i}^{t_{Z}}\right)^{D}-\left(x_{i}^{0}\right)^{D}\right)}{d_{i}^{D}{\underline{t_{Z}}}^{2}}-\frac{2\left(v_{i}^{t_{Z}}+2 v_{i}^{0}\right)}{\underline{t_{Z}}}, \\
& v_{i}^{*}(t)=-\left(\frac{6\left(\left(x_{i}^{t_{Z}}\right)^{D}-\left(x_{i}^{0}\right)^{D}\right)}{d_{i}^{D}{\underline{t_{Z}}}^{3}}-\frac{3\left(v_{i}^{t_{Z}}+v_{i}^{0}\right)}{\underline{t}^{2}}\right) t^{2}+\left(\frac{6\left(\left(x_{i}^{t_{Z}}\right)^{D}-\left(x_{i}^{0}\right)^{D}\right)}{d_{i}^{D}{\underline{t_{Z}}}^{2}}-\frac{2\left(v_{i}^{t_{Z}}+2 v_{i}^{0}\right)}{\underline{t_{Z}}}\right) t+v_{i}^{0}, \\
& \left(x_{i}^{X}\right)^{*}(t)=-\left(\frac{2\left(\left(x_{i}^{t_{Z}}\right)^{D}-\left(x_{i}^{0}\right)^{D}\right)}{d_{i}^{D} \underline{t Z}^{3}}-\frac{\left(v_{i}^{t_{Z}}+v_{i}^{0}\right)}{\underline{t}^{2}}\right) d_{i}^{X} t^{3}+\left(\frac{3\left(\left(x_{i}^{t_{Z}}\right)^{D}-\left(x_{i}^{0}\right)^{D}\right)}{d_{i}^{D} \underline{t \underline{Z}}^{2}}-\frac{\left(v_{i}^{t_{Z}}+2 v_{i}^{0}\right)}{\underline{t_{Z}}}\right) d_{i}^{X} t^{2} \\
& +v_{i}^{0} d_{i}^{X} t+\left(x_{i}^{0}\right)^{X} \text {, } \\
& \left(x_{i}^{Y}\right)^{*}(t)=-\left(\frac{2\left(\left(x_{i}^{t_{Z}}\right)^{D}-\left(x_{i}^{0}\right)^{D}\right)}{d_{i}^{D}{\underline{t_{Z}}}^{3}}-\frac{\left(v_{i}^{t_{Z}}+v_{i}^{0}\right)}{{\underline{t_{Z}}}^{2}}\right) d_{i}^{Y} t^{3}+\left(\frac{3\left(\left(x_{i}^{t_{Z}}\right)^{D}-\left(x_{i}^{0}\right)^{D}\right)}{d_{i}^{D} \underline{t \underline{Z}}^{2}}-\frac{\left(v_{i}^{t_{Z}}+2 v_{i}^{0}\right)}{\underline{t_{Z}}}\right) d_{i}^{Y} t^{2} \\
& +v_{i}^{0} d_{i}^{Y} t+\left(x_{i}^{0}\right)^{Y} \text {, }
\end{aligned}
$$


where $d_{i}^{D}=d_{i}^{X}$ if $d_{i}^{X} \neq 0$ else $d_{i}^{D}=d_{i}^{Y}$ (note that $d_{i}=\left(d_{i}^{X}, d_{i}^{Y}\right)$ is the cartesian direction of aircraft $i$ and cannot be the null vector).

The optimal cost is:

$J^{*}:=J\left(u^{*}\right)=\sum_{i \in I}\left(-\left(\frac{6\left(\left(x_{i}^{t_{Z}}\right)^{D}-\left(x_{i}^{0}\right)^{D}\right)}{d_{i}^{D} \underline{t}^{3}}-\frac{3\left(v_{i}^{t_{Z}}+v_{i}^{0}\right)}{\underline{t}^{2}}\right) t_{f}^{2}+\left(\frac{6\left(\left(x_{i}^{t_{Z}}\right)^{D}-\left(x_{i}^{0}\right)^{D}\right)}{d_{i}^{D} \underline{t \underline{Z}}^{2}}-\frac{2\left(v_{i}^{t_{Z}}+2 v_{i}^{0}\right)}{\underline{t_{Z}}}\right) t_{f}\right)$.

Proof 4 Let us first relax problem $\left(\mathcal{P}_{R_{-} \text {prezone }}\right)$ by discarding bound constraints on $u: \quad \underline{u_{i}} \leq u_{i}(t) \leq \overline{u_{i}}, \quad \forall t \in\left[t_{0}, \underline{t_{Z}}\right] \forall i \in I$.

Note that problem $\left(\mathcal{P}_{R_{-} \text {prezone }}\right)$ corresponds to the sum of $n$ independent subproblems, one for each $i \in I$, as follows:

$$
\left(\mathcal{P}_{\text {prezone }}^{i}\right) \begin{cases}\min _{u_{i}, v_{i}, x_{i}} \int_{0}^{\underline{t_{Z}}} u_{i}^{2}(t) d t & \\ \text { s.t. } & \forall t \in\left[t_{0}, \underline{t_{Z}}\right], \\ \dot{v}_{i}(t)=u_{i}(t) & \forall t \in\left[t_{0}, \underline{t_{Z}}\right] \\ \dot{x}_{i}(t)=v_{i}(t) d_{i} & \\ x_{i}(0)=x_{i}^{0}, \quad v_{i}(0)=v_{i}^{0} & \\ x_{i}\left(\underline{t_{Z}}\right)=x_{i}^{t_{Z}}, \quad v_{i}\left(\underline{t_{Z}}\right)=v_{i}^{\frac{t_{Z}}{2}} & \end{cases}
$$

As mentioned in Remark 4, problem $\left(\mathcal{P}_{\text {postzone }}^{i}\right)$ and problem $\left(\mathcal{P}_{\text {prezone }}^{i}\right)$ only differ because the final position has now to be fixed. Thus, the Hamiltonian of this new system is the same as for problem ( $\left.\mathcal{P}_{\text {postzone }}^{i}\right)$.

Hence, the maximum of the Hamiltonian with respect to $u_{i}$, (by fixing the value $z_{0, i}$ to -1$)$ is the same as for problem $\left(\mathcal{P}_{\text {postzone }}\right): u_{i}^{*}(t)=\underset{u_{i}}{\operatorname{argmax}} H_{i}()=.\frac{z_{3, i}(t)}{2}$.

This result also proves that $u^{*}$ is the unique maximum for problem $\left(\mathcal{P}_{\text {prezone }}^{i}\right)$.

By replacing $u_{i}(t)$ in the differential equations by $\frac{z_{3, i}(t)}{2}$, this yields a system with 6 equations, 6 unknowns, 3 initial conditions and 3 final conditions. Note that we have now two final conditions (coming from the final position at time $\left.\underline{t}_{Z}\right)$. Therefore, the following system of differential equations has to be solved in a different way with respect to what has been done for the postzone, because $\dot{z}_{3, i}$ is no 
more equal to zero and then $z_{3, i}$ and the control $u_{i}^{*}$ is no more constant in $\left[0, \underline{t}_{Z}\right]$ :

$$
\begin{aligned}
& \dot{x}_{i}^{X}(t)=\frac{\partial H_{i}(.)}{\partial z_{1, i}}=v_{i}(t) d_{i}^{X}, \\
& \dot{x}_{i}^{Y}(t)=\frac{\partial H_{i}(.)}{\partial z_{2, i}}=v_{i}(t) d_{i}^{Y}, \\
& \dot{v}_{i}(t)=\frac{\partial H_{i}(.)}{\partial z_{3, i}}=\frac{z_{3, i}(t)}{2}, \\
& \dot{z}_{1, i}(t)=-\frac{\partial H_{i}(.)}{\partial x_{i}^{X}}=0, \\
& \dot{z}_{2, i}(t)=-\frac{\partial H_{i}(.)}{\partial x_{i}^{Y}}=0, \\
& \dot{z}_{3, i}(t)=-\frac{\partial H_{i}(.)}{\partial v_{i}}=-z_{1, i}(t) d_{i}^{X}-z_{2, i}(t) d_{i}^{Y} .
\end{aligned}
$$

The fourth and fifth equations yields that $z_{1, i}^{*}(t)=A$ and $z_{1, i}^{*}(t)=B$, where $A$ and $B$ are two real constants. Hence, the sixth equation gives $\dot{z}_{3, i}(t)=-A d_{i}^{X}-$ $B d_{i}^{Y}$ and then

$$
z_{3, i}(t)=\left(-A d_{i}^{X}-B d_{i}^{Y}\right) t+C,
$$

where $C$ is a new real constant. Note that this provides an optimal control $u^{*}$ which is now affine:

$$
u_{i}^{*}(t)=\frac{\left(-A d_{i}^{X}-B d_{i}^{Y}\right) t+C}{2} .
$$

The third equation yields the optimal velocity $v^{*}$ :

$$
v_{i}^{*}(t)=-\frac{\left(A d_{i}^{X}+B d_{i}^{Y}\right)}{4} t^{2}+\frac{C}{2} t+D,
$$

where $D$ is a new real constant (note that this constant is obviously distinct to the separation distance $D$ and it is just only used in this proof).

The first equation $\dot{x}_{i}^{X}(t)=-\left(\frac{\left(A d_{i}^{X}+B d_{i}^{Y}\right)}{4} t^{2}+\frac{C}{2} t+D\right) d_{i}^{X}$ yields the optimal abscissa position:

$$
\left(x_{i}^{X}\right)^{*}(t)=-\frac{\left(A\left(d_{i}^{X}\right)^{2}+B d_{i}^{X} d_{i}^{Y}\right)}{12} t^{3}+\frac{C}{4} d_{i}^{X} t^{2}+D d_{i}^{X} t+E,
$$

where $E$ is a new real constant. 
Moreover, by introducing a new real constant $F$, and using the second equation, one obtains in the same way the optimal ordinate position:

$$
\left(x_{i}^{Y}\right)^{*}(t)=-\frac{\left(A d_{i}^{X} d_{i}^{Y}+B\left(d_{i}^{Y}\right)^{2}\right)}{12} t^{3}+\frac{C}{4} d_{i}^{Y} t^{2}+D d_{i}^{Y} t+F .
$$

From the 6 initial and final conditions, one can determine the six constant $A, B, C, D, E, F$. Directly, from the initial conditions one obtains $D=v_{i}^{0}, E=$ $\left(x_{i}^{0}\right)^{X}$ and $F=\left(x_{i}^{0}\right)^{Y}$. From the final condition, it remains to solve a linear system of 3 equations and 3 variables $A, B, C$. By defining $Q:=\frac{\left(A d_{i}^{X}+B d_{i}^{Y}\right)}{4}$, one obtains:

$$
\begin{aligned}
& -Q \underline{t}_{Z}^{2}+\frac{C}{2} \underline{t_{Z}}+v_{i}^{0}=v_{i}^{t_{Z}}, \\
& -\frac{1}{3} Q d_{i}^{D} \underline{t_{Z}}{ }^{3}+\frac{C}{4} d_{i}^{D} \underline{t_{Z}}{ }^{2}+v_{i}^{0} d_{i}^{D} \underline{t_{Z}}+\left(x_{i}^{0}\right)^{D}=\left(x_{i}^{t_{Z}}\right)^{D} .
\end{aligned}
$$

Where $d_{i}^{D}$ is equal to $d_{i}^{X}$ or $d_{i}^{Y}$ if and only if they are not null (they cannot be both equal to zero).

Hence, one obtains:

$$
C=\frac{12\left(\left(x_{i}^{t_{Z}}\right)^{D}-\left(x_{i}^{0}\right)^{D}\right)}{d_{i}^{D} \underline{t Z}^{2}}-\frac{4\left(v_{i}^{t_{Z}}+2 v_{i}^{0}\right)}{\underline{t_{Z}}},
$$

and

$$
Q=\frac{6\left(\left(x_{i}^{t_{Z}}\right)^{D}-\left(x_{i}^{0}\right)^{D}\right)}{d_{i}^{D} \underline{t Z}^{3}}-\frac{3\left(v_{i}^{t_{Z}}+v_{i}^{0}\right)}{\underline{t}_{\underline{Z}}^{2}} .
$$

Only these five coefficients are necessary to find all the optimal controls and the associate state variables $u^{*}, v^{*}, x^{*}$ and also the objective value of the cost function $J^{*}$.

Let us consider the bound constraints on $u: \underline{u_{i}} \leq u_{i}(t) \leq \overline{u_{i}}$ for all $i \in I$ and $t \in\left[0, \underline{t_{Z}}\right]:$

$$
\underline{u_{i}} \leq u_{i}^{*}(t)=-2 Q t+\frac{C}{2} \leq \overline{u_{i}}, \forall t \in\left[0, \underline{t_{Z}}\right]
$$

As $u^{*}(t)$ is affine, one has just to check the constraint at time $t=0$ and $t=t_{Z}$ providing: $2 \underline{u_{i}} \leq C \leq 2 \overline{u_{i}}$ and $2 \underline{u_{i}} \leq-4 Q \underline{t_{Z}}+C \leq 2 \overline{u_{i}}$. Then one obtains the two following conditions:

$$
\underline{u_{i}} \leq \frac{C}{2}=\frac{6\left(\left(x_{i}^{t_{Z}}\right)^{D}-\left(x_{i}^{0}\right)^{D}\right)}{d_{i}^{D} \underline{t Z}^{2}}-\frac{2\left(v_{i}^{t_{Z}}+2 v_{i}^{0}\right)}{\underline{t_{Z}}} \leq \overline{u_{i}},
$$


and

$$
\underline{u_{i}} \leq-2 Q \underline{t_{Z}}+\frac{C}{2}=\frac{-6\left(\left(x_{i}^{t_{Z}}\right)^{D}-\left(x_{i}^{0}\right)^{D}\right)}{d_{i}^{D}{\underline{t_{Z}}}^{2}}+\frac{4\left(v_{i}^{t_{Z}}+v_{i}^{0}\right)}{\underline{t_{Z}}} \leq \overline{u_{i}} .
$$

Hence, the unique solution verifying the optimality conditions derived from the PMP is $u^{*}, v^{*}, x^{*}$ if the above conditions (3) and (4) on the control are satisfied. Note that $u_{i}^{*}(t)$ can be also constant in the prezone as in the postzone, if and only if $Q=0$.

The optimal cost function is computed from $u^{*}$ :

$$
J\left(u^{*}\right)=\sum_{i \in I} \int_{0}^{t_{f}}\left(u^{*}\right)^{2} d t=\sum_{i \in I} \int_{0}^{t_{f}}\left(-2 Q t+\frac{C}{2}\right)^{2} d t=\sum_{i \in I}\left(-Q t_{f}^{2}+\frac{C}{2} t_{f}\right) .
$$

Therefore, we proved that from the relaxed problem $\left(\mathcal{P}_{R_{-} \text {prezone }}\right)$, the optimal control $u^{*}$ can be provided using the PMP; the velocity, the position and the final cost can then be derived. However, such a solution exists if the conditions (3) and (4) are satisfied.

Remark 6 Analogously to the case of the postzone, we can observe that the bounds on the control are in practice quite large, and so easily satisfied. It follows that in general, we have a solution of problem $\left(\mathcal{P}_{R_{-} \text {prezone }}\right)$.

Let us now take into account the bounds constraints on $v_{i}(t)$. In this case, we have to consider the following assumption on the shape of the optimal control, which follows directly from Proposition 3 and by the numerical solutions provided in Section 7:

Assumption 1 The optimal control on the time window $\left[0, \underline{t_{Z}}\right]$ has the following shape, for each aircraft $i: u_{i}^{*}(t)$ is affine or $u_{i}^{*}(t)$ is affine from $t=0$ until a critical time that we denote $t_{i}^{c}$, and it is null after. The critical time $t_{i}^{c}$ occurs when the velocity of aircraft $i$ reaches a bound constraint $\underline{v}_{i}$ or $\overline{v_{i}}$. This yields a velocity $v_{i}(t)$ which is quadratic or quadratic and then constant at time $t_{i}^{c}$. Furthermore, let us suppose also that $u^{*}(t)$ remains in its bounds; this implies that the conditions of Proposition 3 are satisfied.

Remark 7 Note that if the velocity $v_{i}(t)$ stays in its bounds for all $t$ in $\left[0, t_{Z}\right]$, then by fixing $t_{i}^{c}=t_{Z}$, one obtains just one shape for the control: affine and null at time $t_{i}^{c}$. 
By decomposing the time interval into three parts $\left[0, \underline{t_{Z}}\right],\left[\underline{t_{Z}}, \overline{t_{Z}}\right]$ and $\left[\overline{t_{Z}}, t_{f}\right]$, by using Propositions 1, 2, 3 and Assumption 1, one can reformulate problem $(\mathcal{P})$ starting from reformulation $\left(\mathcal{P}_{1}\right)$. This provides a new optimization problem with less variables and constraints than problems $(\mathcal{P})$ and $\left(\mathcal{P}_{1}\right)$.

Theorem 2 (Reformulation of problem $(\mathcal{P})$ ) Under Assumption 1, another reformulation of problem $(\mathcal{P})$, with $t_{0}=0$, is:

$$
\begin{aligned}
& \min _{\substack{u, v, x,\left(A, B, t^{c}\right) \in \mathbb{R}^{3 n}}} \sum_{i \in I}\left(\frac{1}{3} A_{i}^{2}\left(t_{i}^{c}\right)^{3}+A_{i} B_{i}\left(t_{i}^{c}\right)^{2}+B_{i}^{2} t_{i}^{c}+\int_{\underline{t_{Z}}}^{\overline{t_{Z}}} u_{i}^{2}(t) d t \quad+\frac{\left(v_{i}^{f}-v_{i}\left(\overline{t_{Z}}\right)\right)^{2}}{t_{f}-\overline{t_{Z}}}\right) \\
& \text { s.t. } \\
& \dot{v}_{i}(t)=u_{i}(t) \\
& \dot{x}_{i}(t)=v_{i}(t) d_{i} \\
& \underline{u_{i}} \leq u_{i}(t) \leq \overline{u_{i}} \\
& \underline{v_{i}} \leq v_{i}(t) \leq \overline{v_{i}} \\
& \underline{u_{i}} \leq \frac{v_{i}^{f}-v_{i}\left(\overline{t_{Z}}\right)}{t_{f}-\overline{t_{Z}}} \leq \overline{u_{i}} \\
& \underline{u_{i}} \leq B_{i} \leq \overline{u_{i}} \\
& \underline{u_{i}} \leq A_{i} t_{i}^{c}+B_{i} \leq \overline{u_{i}} \\
& v_{i}\left(\underline{t_{Z}}\right)=\frac{1}{2} A_{i}\left(t_{i}^{c}\right)^{2}+B_{i} t_{i}^{c}+v_{i}^{0} \\
& x_{i}\left(\underline{t_{Z}}\right)=v_{i}\left(\underline{t_{Z}}\right) d_{i}\left(\underline{t_{Z}}-t_{i}^{c}\right)+\left(\frac{1}{6} A_{i}\left(t_{i}^{c}\right)^{3}+\frac{1}{2} B_{i}\left(t_{i}^{c}\right)^{2}+v_{i}^{0} t_{i}^{c}\right) d_{i}+x_{i}^{0} \\
& {\left[x_{i}\left(\underline{t_{Z}}\right)=\left(\frac{t_{Z}}{2}-\frac{t_{i}^{c}}{3}\right) d_{i} A_{i}\left(t_{i}^{c}\right)^{2}+\left(\underline{t_{Z}}-\frac{t_{i}^{c}}{2}\right) B_{i} d_{i} t_{i}^{c}+\underline{t_{Z}} d_{i} v_{i}^{0}+x_{i}^{0}\right]} \\
& t_{i}^{c}=\min \left\{\underline{t_{Z}}\right\} \cup\left\{t \in\left[0, \underline{t_{Z}}\right], \frac{1}{2} A_{i} t^{2}+B_{i} t+C_{i}=\underline{v_{i}}\right\} \\
& \cup\left\{t \in\left[0, \underline{t_{Z}}\right], \frac{1}{2} A_{i} t^{2}+B_{i} t+C_{i}=\overline{v_{i}}\right\} \\
& x_{i}(0)=x_{i}^{0}, \quad v_{i}(0)=v_{i}^{0} \\
& x_{i}\left(t_{f}\right) \text { free, } \quad v_{i}\left(t_{f}\right)=v_{i}^{f} \\
& \left\|x_{i}(t)-x_{j}(t)\right\|^{2} \geq D^{2}
\end{aligned}
$$

Note that there are major differences between the problem formulations $\mathcal{P}_{1}$ and $\mathcal{P}_{2}$. In the formulation $\mathcal{P}_{2}$, for each aircraft $i$, new real optimization variables 
$A_{i}, B_{i}$, and $t_{i}^{c}$ are introduced. Furthermore, the cost function in $\mathcal{P}_{2}$ is no convex anymore.

Proof 5 The time window $\left[0, t_{f}\right]$ is now decomposed into three parts $\left[t_{0}, t_{Z}\right],\left[\underline{t_{Z}}, \overline{t_{Z}}\right]$ and $\left[\bar{t}_{Z}, t_{f}\right]$, hence problem $(\mathcal{P})$ becomes almost separable. The links occur at time in $t_{Z}$ and also at $\overline{t_{Z}}$. Indeed, the three subproblems can provide disconnected solutions of $u, v$ and $x$, but only at times $\underline{t_{Z}}$ and $\overline{t_{Z}}$.

This new reformulation starts from reformulation $\left(\mathcal{P}_{1}\right)$ yielding the last part of the objective function $\sum_{i \in I} \frac{\left(v_{i}^{f}-v_{i}\left(\overline{t_{Z}}\right)\right)^{2}}{t_{f}-\overline{t_{Z}}}$ and the constraints $\underline{u_{i}} \leq \frac{v_{i}^{f}-v_{i}\left(\overline{t_{Z}}\right)}{t_{f}-\overline{t_{Z}}} \leq$ $\overline{u_{i}}, \forall i \in I$. Note that these are discussed in the proof of Theorem 1. Hence, we have just to demonstrate the first part of this new formulation, from $t=0$ to $\overline{t_{Z}}$.

From Assumption 1, we can directly derive the shape of the solution. Indeed, the control is affine from $t=0$ to $t_{i}^{c}$ :

$$
u_{i}^{*}(t)=A_{i} t+B_{i},
$$

where $A_{i}$ and $B_{i}$ are real constants which are the new unknown of our new formulation. This provides $3 \times n$ new optimization variables: $A_{i}, B_{i}$ and $t_{i}^{c}$. Thus, two new constraints have to be added in order to satisfy the conditions of Proposition 3:

$$
\underline{u_{i}} \leq B_{i} \leq \overline{u_{i}} \text { and } \underline{u_{i}} \leq A_{i} t_{i}^{c}+B_{i} \leq \overline{u_{i}}, \forall i \in I .
$$

From the solution on the optimal control, one can derive the first part of the cost function $J$ :

$$
\int_{0}^{\underline{t_{Z}}}\left(u_{i}^{*}(t)\right)^{2} d t=\int_{0}^{t_{i}^{c}}\left(A_{i} t+B_{i}\right)^{2} d t=\frac{A_{i}^{2}}{3}\left(t_{i}^{c}\right)^{3}+A_{i} B_{i}\left(t_{i}^{c}\right)^{2}+B_{i}^{2} t_{i}^{c} .
$$

Note that $u_{i}^{*}(t)=0$ for all $t$ in $\left[0, t_{i}^{c}\right]$.

From $u_{i}^{*}(t)=A_{i} t+B_{i}$ for all $t$ in $\left[0, t_{i}^{c}\right]$ and zero after, one can provide the velocity:

$$
v_{i}^{*}(t)= \begin{cases}\frac{A_{i}}{2} t^{2}+B_{i} t+v_{i}^{0}, & \forall t \in\left[0, t_{i}^{c}\right] \\ \frac{A_{i}}{2}\left(t_{i}^{c}\right)^{2}+B_{i} t_{i}^{c}+v_{i}^{0}, & \forall t \in\left[t_{i}^{c}, \underline{t_{Z}}\right]\end{cases}
$$

This implies the link between the prezone and the zone at time $\underline{t_{Z}}$ :

$$
v_{i}^{*}\left(\underline{t_{Z}}\right)=v_{i}^{*}\left(t_{i}^{c}\right)=\frac{A_{i}}{2}\left(t_{i}^{c}\right)^{2}+B_{i} t_{i}^{c}+v_{i}^{0}, \forall i \in I .
$$

Now, from the optimal velocity, one can derive the position of aircraft $i$ :

$x_{i}^{*}(t)=t_{i}^{c} \begin{cases}\frac{A_{i}}{6} d_{i} t^{3}+\frac{B_{i}}{2} d_{i} t^{2}+v_{i}^{0} d_{i} t+x_{i}^{0}, & \forall t \in\left[0, t_{i}^{c}\right] \\ \left(\frac{A_{i}}{2}\left(t_{i}^{c}\right)^{2}+B_{i} t_{i}^{c}+v_{i}^{0}\right) d_{i}\left(t-t_{i}^{c}\right)+\frac{A_{i}}{6} d_{i}\left(t_{i}^{c}\right)^{3}+\frac{B_{i}}{2} d_{i}\left(t_{i}^{c}\right)^{2}+v_{i}^{0} d_{i}\left(t_{i}^{c}\right)+x_{i}^{0}, & \forall t \in\left[t_{i}^{c}, \underline{t_{Z}}\right]\end{cases}$ 
Hence, the following constraint at time $\underline{t}_{Z}$ on the position is:

$x_{i}^{*}\left(\underline{t_{Z}}\right)=\left(\frac{A_{i}}{2}\left(t_{i}^{c}\right)^{2}+B_{i} t_{i}^{c}+v_{i}^{0}\right) d_{i}\left(\underline{t_{Z}}-t_{i}^{c}\right)+\frac{A_{i}}{6} d_{i}\left(t_{i}^{c}\right)^{3}+\frac{B_{i}}{2} d_{i}\left(t_{i}^{c}\right)^{2}+v_{i}^{0} d_{i}\left(t_{i}^{c}\right)+x_{i}^{0}$.

Now, it remains to explain the critical time $t_{i}^{c}$. It is defined as the time in $\left[0, t_{Z}\right]$ when the velocity of aircraft $i$ reaches for the first time a limit $\underline{v_{i}}$ or $\overline{v_{i}}$. Note that if the velocity stays in $\left[v_{i}, \overline{v_{i}}\right]$, then $t_{i}^{c}$ has to be fixed to $t_{Z}$. Therefore, the following condition involving $t_{i}^{c}, \bar{A}_{i}$ and $B_{i}$ holds:

$t_{i}^{c}=\min \left\{\underline{t_{Z}}\right\} \cup\left\{t \in\left[0, \underline{t_{Z}}\right], \frac{1}{2} A_{i} t^{2}+B_{i} t+C_{i}=\underline{v_{i}}\right\} \cup\left\{t \in\left[0, \underline{t_{Z}}\right], \frac{1}{2} A_{i} t^{2}+B_{i} t+C_{i}=\overline{v_{i}}\right\}$

That concludes the proof.

Note that the control $u$ can be discontinuous, because it is possible for an aircraft to roughly change its acceleration. Therefore, $u_{i}$ can be discontinuous at time $\overline{t_{Z}}$ but also at times $t_{i}^{c}$ and $t_{Z}$; indeed, $u_{i}$ has just to be integrable over $\left[0, t_{i}^{c}\right]$, $\left[t_{i}^{c}, \underline{t_{Z}}\right],\left[t_{Z}, \overline{t_{Z}}\right]$ and $\left[\overline{t_{Z}}, t_{f}\right]$. For the velocity $v$ and the position $x$, one must keep the continuity of the solution at times $t_{i}^{c}, \underline{t_{Z}}$ and $\overline{t_{Z}}$.

Remark 8 The new formulation $\left(\mathcal{P}_{2}\right)$ only provides solutions for all $t$ in $\left[t_{Z}, \overline{t_{Z}}\right]$ (if such an optimal solution exists). All the remaining solutions, for all time $t$ in $\left[0, t_{Z}\right]$, have been developed in the proof of Theorem 2. The last part of the solutions for a time $t$ in $\left[\overline{t_{Z}}, t_{f}\right]$ are given in Proposition 2.

\section{Applying the direct shooting method}

Let us now focus on the numerical solution of Problem $(\mathcal{P})$ and its two reformulations $\left(\mathcal{P}_{1}\right)$ and $\left(\mathcal{P}_{2}\right)$. In all these three cases, we have to solve an optimal control 
problem in a time window $\left[t_{a}, t_{b}\right]$, with $t_{0} \leq t_{a} \leq t_{b} \leq t_{f}$, of the following type:

$$
(\tilde{\mathcal{P}})\left\{\begin{array}{lr}
\min _{u, v, x} \sum_{i \in I} \int_{t_{a}}^{t_{b}} u_{i}^{2}(t) d t & \\
\text { s.t. } & \forall t \in\left[t_{a}, t_{b}\right], \forall i \in I \\
\dot{v}_{i}(t)=u_{i}(t) & \forall t \in\left[t_{a}, t_{b}\right], \forall i \in I \\
\dot{x}_{i}(t)=v_{i}(t) d_{i} & \forall t \in\left[t_{a}, t_{b}\right], \forall i \in I \\
u_{i} \leq u_{i}(t) \leq \overline{u_{i}} & \forall t \in\left[t_{a}, t_{b}\right], \forall i \in I \\
\underline{v_{i}} \leq v_{i}(t) \leq \overline{v_{i}} & \forall i \in I \\
x_{i}\left(t_{a}\right)=x_{i}^{a}, \quad v_{i}\left(t_{a}\right)=v_{i}^{a} & \forall i \in I \\
x_{i}\left(t_{b}\right) \text { free }, \quad v_{i}\left(t_{b}\right)=v_{i}^{b} & \forall t \in\left[t_{a}, t_{b}\right], \\
\left\|x_{i}(t)-x_{j}(t)\right\|^{2} \geq D^{2} & \forall(i, j) \in I^{2}, i<j
\end{array}\right.
$$

Problem $(\tilde{\mathcal{P}})$ corresponds to Problem $(\mathcal{P})$ when $t_{a}=t_{0}$ and $t_{b}=t_{f}$. In the case when we consider the problem decomposition based on the postzone, then $t_{a}=t_{0}$ and $t_{b}=\overline{t_{Z}}$; finally in the case when we consider the problem decomposition based on the prezone, then $t_{a}=t_{Z}$ and $t_{b}=\overline{t_{Z}}$.

By applying the direct shooting method to the optimal control problem $(\tilde{\mathcal{P}})$, the ordinary differential equations are computationally treated by discretizing the variables appearing in the equations with respect to the time. Numerical integrators (for example, Euler-type integrators) are used to approximate the differential equations.

By discretizing the time in $\left[t_{a}, t_{b}\right]$ into $N$ steps $\left(h=\frac{t_{b}-t_{a}}{N}\right)$, and then by discretizing the controls $u_{i}$ and the state variables $v_{i}, x_{i}$ (for all aircraft $i$ ), we obtain the following NLP problem: 


$$
\left(\tilde{P}^{d i s c}\right) \begin{cases}\min _{(x, v, u)} h \sum_{i \in I} \sum_{k \in K}\left(u_{i}^{(k)}\right)^{2} & \\ \text { s.t. } & \forall k \in K, \forall i \in I \\ v_{i}^{(k+1)}=\operatorname{NUM}_{v_{i}}\left(u_{i}^{(k)}\right) & \forall k \in K, \forall i \in I \\ x_{i}^{(k+1)}=\operatorname{NUM}_{x_{i}}\left(v_{i}^{(k)}\right) & \forall k \in K, \forall i \in I \\ \underline{u_{i}} \leq u_{i}^{(k)} \leq \overline{u_{i}} & \forall k \in K, \forall i \in I \\ \underline{v_{i}} \leq v_{i}^{(k)} \leq \overline{v_{i}} & \forall i \in I \\ v_{i}^{(0)}=v_{i}^{a} & \forall i \in I \\ x_{i}^{(0)}=x_{i}^{a} & \forall i \in I \\ v_{i}^{(N)}=v_{i}^{b} & \forall k \in K, \forall i<j,(i, j) \in I^{2} \\ \left\|x_{i}^{(k)}-x_{j}^{(k)}\right\|^{2} \geq D^{2}\end{cases}
$$

where NUM is a numerical integrator depending on the state variables, and $u_{i}^{(k)}, v_{i}^{(k)}$ and $x_{i}^{(k)}$ denote the values of the acceleration, velocity and respectively position at time $t_{k}=k h$. Variables are indexed on the set $K=\{0, \ldots, N-1\}$.

From problem $\left(\mathcal{P}_{1}\right)$, we obtain the following discretized problem:

$$
\left(\tilde{P}_{1}^{d i s c}\right)\left\{\begin{array}{cc}
\min _{(x, v, u)} h \sum_{i \in I} \sum_{k \in K}\left(u_{i}^{(k)}\right)^{2}+\sum_{i \in I} \frac{\left(v_{i}^{f}-v_{i}^{(N)}\right)^{2}}{t_{f}-\overline{t_{Z}}} \\
\text { s.t. } \quad \forall k \in K, \forall i \in I \\
v_{i}^{(k+1)}=\operatorname{NUM}_{v_{i}}\left(u_{i}^{(k)}\right) & \forall k \in K, \forall i \in I \\
x_{i}^{(k+1)}=\operatorname{NUM}_{x_{i}}\left(v_{i}^{(k)}\right) & \forall k \in K, \forall i \in I \\
\underline{u_{i}} \leq u_{i}^{(k)} \leq \overline{u_{i}} & \forall k \in K, \forall i \in I \\
\underline{v_{i}} \leq v_{i}^{(k)} \leq \overline{v_{i}} & \forall i \in I \\
\underline{u_{i}} \leq \frac{v_{i}^{f}-v_{i}^{(N)}}{t_{f}-\overline{t_{Z}}} \leq \overline{u_{i}} & \forall i \in I \\
v_{i}^{(0)}=v_{i}^{0} & \forall i \in I \\
x_{i}^{(0)}=x_{i}^{0} & \forall k \in K, \forall i<j,(i, j) \in I^{2} \\
v_{i}^{(N)}=v_{i}^{\overline{Z_{Z}}} & \forall i \in x_{j} \\
\left\|x_{i}^{(k)}-x_{j}^{(k)}\right\|^{2} \geq D^{2} &
\end{array}\right.
$$


From problem $\left(\mathcal{P}_{2}\right)$, we obtain the following discretized problem:

$$
\begin{aligned}
& \min _{\substack{u, v, x,\left(A, B, t^{c}\right) \in\left(\mathbb{R}^{n}\right)^{3}}} \sum_{i \in I}\left(\frac{1}{3} A_{i}^{2}\left(t_{i}^{c}\right)^{3}+A_{i} B_{i}\left(t_{i}^{c}\right)^{2}+B_{i}^{2} t_{i}^{c}+h \sum_{k \in K}\left(u_{i}^{(k)}\right)^{2}+\frac{\left(v_{i}^{f}-v_{i}^{(N)}\right)^{2}}{t_{f}-\overline{t_{Z}}}\right) \\
& \text { s.t. } \\
& v_{i}^{(k+1)}=\mathrm{NUM}_{v_{i}}\left(u_{i}^{(k)}\right) \quad \forall k \in K, \forall i \in I \\
& x_{i}^{(k+1)}=\operatorname{NUM}_{x_{i}}\left(v_{i}^{(k)}\right) \quad \forall k \in K, \forall i \in I \\
& \underline{u_{i}} \leq u_{i}^{(k)} \leq \overline{u_{i}} \quad \forall k \in K, \forall i \in I \\
& \underline{v_{i}} \leq v_{i}^{(k)} \leq \overline{v_{i}} \quad \forall k \in K, \forall i \in I \\
& \underline{u_{i}} \leq \frac{v_{i}^{f}-v_{i}^{(N)}}{t_{f}-\overline{t_{Z}}} \leq \overline{u_{i}} \quad \forall i \in I \\
& \left(\tilde{P}_{2}^{\text {disc }}\right) \begin{cases}u_{i} \leq B_{i} \leq \overline{u_{i}} & \forall i \in I\end{cases} \\
& \underline{u_{i}} \leq A_{i} t_{i}^{c}+B_{i} \leq \overline{u_{i}} \quad \forall i \in I \\
& t_{i}^{c}=\min \left\{\underline{t_{Z}}\right\} \cup\left\{t \in\left[0, \underline{t_{Z}}\right], \frac{1}{2} A_{i} t^{2}+B_{i} t+C_{i}=\underline{v_{i}}\right\} \\
& \cup\left\{t \in\left[0, \underline{t_{Z}}\right], \frac{1}{2} A_{i} t^{2}+B_{i} t+C_{i}=\overline{v_{i}}\right\} \quad \forall i \in I \\
& v_{i}^{(0)}=\frac{1}{2} A_{i}\left(t_{i}^{c}\right)^{2}+B_{i} t_{i}^{c}+v_{i}^{0} \quad \forall i \in I \\
& x_{i}^{(0)}=v_{i}\left(\underline{t_{Z}}\right) d_{i}\left(\underline{t_{Z}}-t_{i}^{c}\right)+\left(\frac{1}{6} A_{i}\left(t_{i}^{c}\right)^{3}+\frac{1}{2} B_{i}\left(t_{i}^{c}\right)^{2}+v_{i}^{0} t_{i}^{c}\right) d_{i}+x_{i}^{0} \quad \forall i \in I \\
& v_{i}^{(N)}=v_{i}^{\overline{t_{Z}}} \quad \forall i \in I \\
& \left\|x_{i}^{(k)}-x_{j}^{(k)}\right\|^{2} \geq D^{2} \quad \forall k \in K, \forall i<j,(i, j) \in I^{2}
\end{aligned}
$$

By considering $h$ fixed, we have $N$ discretization steps for $\left(\tilde{P}^{d i s c}\right)$ with $t_{a}=t_{0}$ and $t_{b}=t_{f}$, and respectively $N_{1}$ and $N_{2}$ for problems $\left(\tilde{P}_{1}^{d i s c}\right)$ and $\left(\tilde{P}_{2}^{\text {disc }}\right)$; note that $N \geq N_{1} \geq N_{2}$. Hence, for $\left(\tilde{P}^{\text {disc }}\right)$ with $t_{a}=t_{0}$ and $t_{b}=t_{f}$, we have $4 n N$ variables, $N\left(\frac{n(n-1)}{2}+3 n\right)+n$ equality and inequality constraints and $4 n N$ bound constraints, for $\left(\tilde{P}_{1}^{d i s c}\right), 4 n N_{1}$ variables, $N_{1}\left(\frac{n(n-1)}{2}+3 n\right)+n$ equality and inequality constraints and $4 n N_{1}+n$ bound constraints and for $\left(\tilde{P}_{2}^{\text {disc }}\right), 4 n N_{2}+3 n$ variables, $N_{1}\left(\frac{n(n-1)}{2}+4 n\right)+3 n$ equality and inequality constraints and $4 n N_{1}+3 n$ bound constraints.

Note that we can alternatively use a semi-direct shooting method on $(\tilde{\mathcal{P}})$ where 
the only variables are the discretized controls $u_{i}^{(k)}$ and the discretized state variables are computed from $u_{i}^{(k)}$ through the equality constraints. This yields more compact formulations with $n N$ variables, $N\left(\frac{n(n-1)}{2}\right)+n$ equality and inequality constraints and $4 n N$ bound constraints. Nevertheless, we show in [12] that the direct shooting method is more efficient on the considered problem. Therefore in the following we use the direct shooting method for our computational tests.

\section{Computational Results}

In this section, we discuss numerical results obtained by applying the proposed optimal control-based approaches to solve the aircraft conflict avoidance problem. Computational experiments were carried out using the AMPL [18] modelling environment and the Interior Point method-based solver IPOPT [25] for large-scale nonlinear problems. We implemented our approach implying the application of the PMP in the prezone in $\mathrm{C}$ programming language, as the computation of $t^{c}$ induces logical expressions to be verified, that makes an AMPL implementation (easily applied in the two cases other than the prezone) not much performing. All the numerical tests were performed on a $2.66 \mathrm{GHz}$ Intel Xeon (octo-core) processor with $32 \mathrm{~GB}$ of RAM and Linux Operating System.

Test problem instances are generated in the following way. Aircraft are placed on a circle, flying with a heading pointing toward the center of the circle but deviated with an angle belonging to $[-\pi / 6,+\pi / 6]$, see Figure 5 for an illustration. The aircraft trajectories are represented as straight lines in $\mathbb{R}^{2}$. The horizontal separation norm is 5NM. For each aircraft $i$, its initial (nominal) velocity $v_{i}^{t_{0}}$ is either 400 or $447 \mathrm{NM} / \mathrm{h}$, or belongs to the interval $[400 ; 450]$. These values correspond to common aircraft speeds. The tight bounds imposed on velocity values are due to operational reasons, and follow the directives of the ERASMUS project [4] to perform a subliminal velocity control. Namely, we consider:

- $\left[v_{i}^{t_{0}}-6 \% v_{i}^{t_{0}}, v_{i}^{t_{0}}+3 \% v_{i}^{t_{0}}\right]$ for the 'weak' ERASMUS regulation;

- $\left[v_{i}^{t_{0}}-12 \% v_{i}^{t_{0}}, v_{i}^{t_{0}}+6 \% v_{i}^{t_{0}}\right]$ for the 'strong' ERASMUS regulation;

- $\left[v_{i}^{t_{0}}-10 \% v_{i}^{t_{0}}, v_{i}^{t_{0}}+10 \% v_{i}^{t_{0}}\right]$ for a symmetric-range regulation.

The bounds imposed on aircraft accelerations are based on the Eurocontrol's Base of Aircraft Data (BADA) [17], and have values $\overline{u_{i}}=-u_{i}=4000 \mathrm{NM} / \mathrm{h}^{2}$.

A time window $\left[t_{0}, t_{f}\right]$ with $t_{0}=0$ and $t_{f}=1 \mathrm{~h}$ is considered to issue separation maneuvers (acting on velocities), ensuring separation throughout all the time window. At final time $t_{f}$, aircraft are expected to return to their nominal velocities $v_{i}^{t_{0}}$ (terminal conditions in our optimal control model). 
Table 1 summarizes the characteristics of the considered problem instances: number of aircraft $n$, number of initial potential conflicts, number of initial conflicts actually occurring unless speed changes are performed (potential concourses), percentage of time spent in the zone (see Section 3), nominal velocities (in NM/h), allowed percentage of speed change (e.g., with the weak ERASMUS regulation, from $-6 \%$ to $+3 \%$ ), and sum of all initial potential conflict times.

In our numerical experiments, the time discretization step is equal to $h=15^{\prime \prime}$. The starting points are deduced from the trivial controls, in the sense that all the state variables (velocities and positions) are computed from command (acceleration) values equal to 0 . A complete descriptions of these 30 instances can be found in [11].

In Tables 2, 3 and 4, we present the results obtained applying the proposed optimal control approaches, presented in Sections 4, 5 and 6.

In these tables, for each problem instance, we report the number of variables, the number of constraints, the total number of iterations and the number of function evaluations carried out by the NLP solver (IPOPT), the computed objective function value and the computing time.

Table 2 reports the results obtained applying the direct shooting method on the whole time window $\left[0, t_{f}\right]$, while the results in Table 3 and the results in Table 4 are obtained applying such method respectively only in the time interval $\left[0, \overline{t_{Z}}\right]$ (i.e., from the initial time to the end of the zone), then computing the PMP-based solution in the postzone, and only in the time interval $\left[t_{Z}, \overline{t_{Z}}\right]$ (i.e., in the zone), then computing the PMP-based solution in the prezone as well as in the postzone.

Let us first consider Table 2. We are able to compute an optimal solution for 29 problem instances out of 30 (pb16 for which the maximum number of iterations is reached). As expected, the computing time increases with the size of the problem and its complexity, that is mainly related to the number of potential conflicts and concourses. Applying the direct method on the whole time window yields in two cases to computing times that exceed 300 seconds.

For the sake of illustration, Figure 5 represents the $\mathbb{R}^{2}$-trajectory configuration for the 13-aircraft conflict avoidance problem pb29. For the same problem instance, Figure 6 displays the optimal velocity solution curves.

Combining the direct method to the PMP in the postzone (Table 3), computing times are drastically reduced. As expected, the benefit is directly related to the size of the time window during which the direct method is applied, and thereby to the size of the zone: the more the zone is small, the more the PMP is applied on a larger time interval, thus yielding a reduction in the total computing time. More specifically, the size of the zone is related to the number of variables and constraints 


\begin{tabular}{|c|c|c|c|c|c|c|c|c|c|c|c|}
\hline \multirow{2}{*}{$\begin{array}{l}\text { pb. } \\
\text { ID }\end{array}$} & \multirow{2}{*}{$\begin{array}{l}\text { nb. of } \\
\text { aircr. }\end{array}$} & \multirow{2}{*}{$\begin{array}{l}\text { nb. of } \\
\text { confl. }\end{array}$} & \multirow{2}{*}{$\begin{array}{l}\text { nb. of } \\
\text { conc. }\end{array}$} & \multirow{2}{*}{$\begin{array}{l}\text { ZONE time } \\
\% \text { w.r.t. } 1 \mathrm{~h} \\
\end{array}$} & \multicolumn{3}{|c|}{ nominal velocity $(\mathrm{NM} / \mathrm{h})$} & \multicolumn{3}{|c|}{ velocity bound percents } & \multirow{2}{*}{$\begin{array}{l}\text { conflict times } \\
\text { sum (seconds) }\end{array}$} \\
\hline & & & & & 400 & 447 & {$[400 ; 450]$} & {$[-6 ; 3]$} & {$[-12 ; 6]$} & {$[-10 ; 10]$} & \\
\hline pb01 & 3 & 2 & 3 & 13 & $\checkmark$ & & & & & $\checkmark$ & 61.0 \\
\hline pb02 & 3 & 2 & 3 & 16 & $\checkmark$ & & & & & $\checkmark$ & 50.5 \\
\hline pb03 & 3 & 2 & 2 & 11 & $\checkmark$ & & & $\checkmark$ & & & 73.3 \\
\hline pb04 & 4 & 3 & 3 & 16 & & & $\checkmark$ & & & $\checkmark$ & 150.0 \\
\hline pb05 & 4 & 3 & 3 & 16 & & & $\checkmark$ & & $\checkmark$ & & 102.2 \\
\hline pb06 & 5 & 4 & 5 & 26 & & $\checkmark$ & & & & $\checkmark$ & 206.1 \\
\hline pb07 & 5 & 4 & 5 & 26 & & & $\checkmark$ & & & $\checkmark$ & 225.0 \\
\hline pb08 & 6 & 4 & 6 & 41 & $\checkmark$ & & & $\checkmark$ & & & 315.7 \\
\hline pb09 & 6 & 4 & 5 & 31 & & & $\checkmark$ & & $\checkmark$ & & 207.9 \\
\hline pb10 & 6 & 4 & 5 & 15 & & & $\checkmark$ & $\checkmark$ & & & 225.0 \\
\hline pb11 & 7 & 4 & 11 & 41 & $\checkmark$ & & & & $\checkmark$ & & 416.4 \\
\hline pb12 & 7 & 4 & 11 & 36 & & $\checkmark$ & & & $\checkmark$ & & 372.6 \\
\hline pb13 & 7 & 4 & 9 & 26 & & & $\checkmark$ & & & $\checkmark$ & 291.6 \\
\hline pb14 & 8 & 4 & 7 & 41 & $\checkmark$ & & & & $\checkmark$ & & 346.2 \\
\hline pb15 & 8 & 4 & 5 & 53 & $\checkmark$ & & & $\checkmark$ & & & 236.6 \\
\hline pb16 & 8 & 6 & 10 & 16 & $\checkmark$ & & & $\checkmark$ & & & 411.2 \\
\hline pb17 & 9 & 5 & 13 & 35 & $\checkmark$ & & & & $\checkmark$ & & 357.0 \\
\hline pb18 & 9 & 5 & 13 & 31 & & $\checkmark$ & & & $\checkmark$ & & 319.5 \\
\hline pb19 & 9 & 5 & 13 & 31 & & $\checkmark$ & & & & $\checkmark$ & 319.5 \\
\hline pb20 & 10 & 5 & 5 & 31 & & $\checkmark$ & & & $\checkmark$ & & 232.0 \\
\hline pb21 & 10 & 5 & 5 & 31 & & $\checkmark$ & & & & $\checkmark$ & 232.0 \\
\hline pb22 & 10 & 5 & 5 & 35 & $\checkmark$ & & & & $\checkmark$ & & 259.2 \\
\hline pb23 & 11 & 6 & 17 & 45 & $\checkmark$ & & & $\checkmark$ & & & 521.1 \\
\hline pb24 & 11 & 5 & 7 & 54 & $\checkmark$ & & & $\checkmark$ & & & 259.1 \\
\hline pb25 & 11 & 6 & 15 & 51 & & & $\checkmark$ & & $\checkmark$ & & 320.4 \\
\hline pb26 & 12 & 6 & 12 & 32 & $\checkmark$ & & & $\checkmark$ & & & 643.0 \\
\hline pb27 & 12 & 6 & 15 & 46 & & $\checkmark$ & & $\checkmark$ & & & 472.2 \\
\hline pb28 & 12 & 6 & 12 & 29 & & $\checkmark$ & & $\checkmark$ & & & 575.4 \\
\hline pb29 & 13 & 9 & 14 & 67 & & & $\checkmark$ & $\checkmark$ & & & 1294.0 \\
\hline pb30 & 13 & 6 & 14 & 59 & & $\checkmark$ & & $\checkmark$ & & & 418.3 \\
\hline
\end{tabular}

Table 1: Data instances: problem names, number of aircraft, number of initial potential conflicts, number of initial potential concourses, time spent in the zone, nominal velocity, percentages of speed changes, and sum of durations of initial potential conflict. 


\begin{tabular}{c|rr|rr|rr}
\hline pb. & nb. of & nb. of & nb. of & nb. of & obj. & cpu time \\
ID & var. & constr. & it. & eval. & value & (seconds) \\
\hline pb01 & 2898 & 5781 & 19 & 31 & 78.212 & 0.892 \\
pb02 & 2898 & 5781 & 37 & 234 & 145.547 & 1.828 \\
pb03 & 2898 & 5781 & 23 & 137 & 912.703 & 0.976 \\
pb04 & 3864 & 8190 & 33 & 42 & 1073.489 & 1.860 \\
pb05 & 3864 & 8190 & 97 & 551 & 648.361 & 5.220 \\
pb06 & 4830 & 10840 & 138 & 957 & 3161.899 & 10.888 \\
pb07 & 4830 & 10840 & 45 & 121 & 948.502 & 3.720 \\
pb08 & 5796 & 13731 & 136 & 158 & 1271.155 & 18.804 \\
pb09 & 5796 & 13731 & 31 & 116 & 335.791 & 3.840 \\
pb10 & 5796 & 13731 & 63 & 79 & 1675.380 & 6.960 \\
pb11 & 6762 & 16863 & 41 & 75 & 601.908 & 6.864 \\
pb12 & 6762 & 16863 & 52 & 99 & 796.172 & 8.968 \\
pb13 & 6762 & 16863 & 26 & 141 & 714.660 & 4.664 \\
pb14 & 7728 & 20236 & 68 & 139 & 931.898 & 13.232 \\
pb15 & 7728 & 20236 & 41 & 158 & 1857.248 & 9.760 \\
pb16 & 7728 & 20236 & 3000 & 4238 & 118446.731 & 592.500 \\
pb17 & 8694 & 23850 & 26 & 105 & 646.542 & 8.192 \\
pb18 & 8694 & 23850 & 95 & 581 & 814.456 & 30.272 \\
pb19 & 8694 & 23850 & 48 & 212 & 814.456 & 15.332 \\
pb20 & 9660 & 27705 & 847 & 8941 & 6130.617 & 339.148 \\
pb21 & 9660 & 27705 & 112 & 974 & 6130.617 & 43.396 \\
pb22 & 9660 & 27705 & 119 & 1040 & 4921.333 & 43.676 \\
pb23 & 10626 & 31801 & 105 & 220 & 5885.763 & 64.260 \\
pb24 & 10626 & 31801 & 57 & 240 & 1612.852 & 33.076 \\
pb25 & 10626 & 31801 & 51 & 135 & 2675.255 & 31.464 \\
pb26 & 11592 & 36138 & 161 & 841 & 2412.280 & 122.644 \\
pb27 & 11592 & 36138 & 152 & 1107 & 6134.474 & 116.588 \\
pb28 & 11592 & 36138 & 137 & 329 & 3065.079 & 105.488 \\
pb29 & 12558 & 40716 & 78 & 337 & 4930.398 & 79.176 \\
pb30 & 12558 & 40716 & 136 & 749 & 3025.378 & 137.604 \\
\hline & & & & & & \\
\end{tabular}

Table 2: Numerical results obtained by applying the direct solution method on the whole time window: number of variables, number of constraints, number of iterations, number of funtion evaluations, objective value, computing time. 
of the NLP problem to be solved with the direct method. The computed solution is the same in most of the cases, except for two problem instances: pb16 which is again not solved and pb08. Remark that computed solutions are local optima and so, different minima can be provided.

Applying the PMP also in the prezone (Table 4) requires a larger computational effort compared to the application of the PMP in the postzone only, yielding larger computing times. This behaviour is related to the increased complexity of the problem to be solved when the PMP is applied in the prezone, as discussed in Section5. As a consequence, we are not able, in general, to reduce furtherly the computing time with respect to the case of the PMP on the postzone only, and computing times in some cases are even larger than those required by the application of the direct method on the whole time window (Table 2). On the other hand, we remark a better quality of the solution in most cases, as expected. Remark also that pb16 is now solved to optimality. Furthermore, our assumptions of the form of the solution are confirmed by the experimental results.

In order to more clearly compare the results obtained for our three proposed approaches, we make use of the performance profiles of Dolan and Moré [15]. We recall that when using performance profiles, for $\tau \geq 0$ on the x-axis, one can read on the y-axis the fraction $\rho_{s}(\tau)$ of problems for which the performance of a given algorithm is within a factor $2^{\tau}$ of the best one. In our case, Figure 3 displays a performance profile comparing the results in terms of objective function values, and Figure 4 displays a performance profile comparing the results in terms of computing time. Figure 3 shows that the approach based on the PMP applied in both the prezone and the postzone ensures the most accurate computation of the solutions. Figure 4 clearly highlights the benefit, in terms of CPU time, of using the approach based on the PMP applied in the postzone only.

To summarize, the case of PMP in the postzone appears to offer the best tradeoff between ease of implementation and quality of solution, while being very efficient in terms of computing time.

\section{Conclusion}

In this paper, we consider the problem of aircraft conflict avoidance using only (subliminal) velocity regulation, keeping the $\mathbb{R}^{3}$-trajectories unchanged. We propose a decomposition tailored to this problem into specific zones with respect to the pairwise aircraft separation constraints. We show that into two of these zones conflicts cannot occur, so separation constraints can be neglected, and we can provide analytical solutions by using the PMP, where the criterion is the sum of squared accelerations. This leads to two new formulations, $(\mathcal{P}$ postzone $)$ and $(\mathcal{P}$ prezone $)$, 


\begin{tabular}{c|rr|rr|rr}
\hline pb. & $\begin{array}{r}\text { nb. of } \\
\text { var. }\end{array}$ & $\begin{array}{rlr}\text { constr. } \\
\text { ID }\end{array}$ & $\begin{aligned} \text { nb. of } \\
\text { it. }\end{aligned}$ & $\begin{array}{r}\text { nb. of } \\
\text { eval. }\end{array}$ & $\begin{array}{r}\text { obj. } \\
\text { value }\end{array}$ & $\begin{array}{r}\text { cpu time } \\
\text { (seconds) }\end{array}$ \\
\hline pb01 & 1698 & 3057 & 45 & 48 & 78.212 & 0.924 \\
pb02 & 1674 & 3033 & 27 & 59 & 145.547 & 0.540 \\
pb03 & 1686 & 3018 & 29 & 44 & 912.703 & 0.556 \\
pb04 & 2280 & 4208 & 45 & 47 & 1073.489 & 1.232 \\
pb05 & 2200 & 4068 & 60 & 90 & 648.361 & 1.524 \\
pb06 & 2990 & 5845 & 89 & 276 & 3161.899 & 3.520 \\
pb07 & 2990 & 5845 & 59 & 98 & 948.502 & 2.428 \\
pb08 & 3492 & 7593 & 77 & 96 & 2839.976 & 4.548 \\
pb09 & 3996 & 8100 & 56 & 103 & 335.791 & 3.676 \\
pb10 & 3156 & 6060 & 94 & 102 & 1675.380 & 3.956 \\
pb11 & 5138 & 11074 & 67 & 99 & 601.908 & 7.236 \\
pb12 & 4578 & 9863 & 90 & 141 & 796.172 & 8.244 \\
pb13 & 4130 & 8533 & 63 & 242 & 714.660 & 4.940 \\
pb14 & 6320 & 13844 & 114 & 172 & 931.898 & 16.160 \\
pb15 & 6384 & 14768 & 118 & 208 & 1857.248 & 17.888 \\
pb16 & 4592 & 9112 & 3000 & 3907 & 123212.425 & 195.508 \\
pb17 & 5598 & 12843 & 47 & 65 & 646.542 & 6.408 \\
pb18 & 5022 & 11511 & 50 & 76 & 814.456 & 6.128 \\
pb19 & 4914 & 11286 & 45 & 81 & 814.456 & 5.536 \\
pb20 & 5820 & 13640 & 54 & 150 & 6130.617 & 7.720 \\
pb21 & 5700 & 13385 & 37 & 52 & 6130.617 & 4.748 \\
pb22 & 6500 & 15235 & 49 & 69 & 4921.333 & 7.292 \\
pb23 & 7062 & 18348 & 210 & 875 & 5885.763 & 62.988 \\
pb24 & 8426 & 22000 & 197 & 1259 & 1612.852 & 79.524 \\
pb25 & 7678 & 20251 & 99 & 197 & 2675.255 & 36.728 \\
pb26 & 7224 & 17856 & 109 & 194 & 2412.280 & 33.808 \\
pb27 & 7176 & 19884 & 146 & 808 & 6134.474 & 57.076 \\
pb28 & 6456 & 15918 & 133 & 247 & 3065.079 & 36.372 \\
pb29 & 10478 & 31057 & 136 & 235 & 4930.398 & 103.036 \\
pb30 & 9438 & 27755 & 146 & 651 & 3025.378 & 94.232 \\
\hline & & & & & &
\end{tabular}

Table 3: Numerical results obtained by applying the direct solution method from $t_{0}$ to the end of the zone, followed by the PMP-based solution in the postzone: number of variables, number of constraints, number of iterations, number of funtion evaluations, objective value, computing time. 


\begin{tabular}{|c|c|c|c|c|c|c|}
\hline $\begin{array}{l}\text { pb. } \\
\text { ID }\end{array}$ & $\begin{array}{l}\text { nb. of } \\
\text { var. }\end{array}$ & $\begin{array}{r}\text { nb. of } \\
\text { constr. }\end{array}$ & $\begin{array}{l}\text { nb. of } \\
\text { it. }\end{array}$ & $\begin{array}{r}\text { nb. of } \\
\text { eval. }\end{array}$ & $\begin{array}{r}\text { obj. } \\
\text { value }\end{array}$ & $\begin{array}{l}\text { cpu time } \\
\text { (seconds) }\end{array}$ \\
\hline pb01 & 105 & 204 & 35 & 40 & 77.373 & 0.184 \\
\hline pb02 & 123 & 240 & 24 & 29 & 143.983 & 0.180 \\
\hline pb03 & 87 & 168 & 35 & 53 & 903.409 & 0.128 \\
\hline pb04 & 164 & 398 & 42 & 61 & 1060.880 & 0.512 \\
\hline pb05 & 164 & 398 & 30 & 43 & 641.733 & 0.336 \\
\hline pb06 & 325 & 955 & 48 & 70 & 3122.111 & 2.616 \\
\hline pb07 & 325 & 955 & 33 & 34 & 938.711 & 1.696 \\
\hline pb08 & 612 & 2112 & 64 & 91 & 1756.963 & 15.348 \\
\hline pb09 & 462 & 1587 & 85 & 120 & 331.512 & 11.968 \\
\hline pb10 & 234 & 789 & 110 & 247 & 1656.187 & 3.068 \\
\hline pb11 & 714 & 2814 & 50 & 58 & 597.113 & 19.208 \\
\hline pb12 & 637 & 2506 & 80 & 100 & 788.753 & 26.520 \\
\hline pb13 & 455 & 1778 & 67 & 84 & 706.879 & 8.716 \\
\hline pb14 & 816 & 3616 & 121 & 174 & 1210.623 & 63.448 \\
\hline pb15 & 1048 & 4660 & 111 & 158 & 1833.433 & 131.428 \\
\hline pb16 & 328 & 1420 & 239 & 543 & 3238.583 & 10.300 \\
\hline pb17 & 783 & 3843 & 99 & 130 & 639.962 & 52.548 \\
\hline pb18 & 702 & 3438 & 94 & 114 & 805.225 & 36.196 \\
\hline pb19 & 693 & 3393 & 86 & 104 & 805.356 & 32.976 \\
\hline pb20 & 790 & 4255 & 86 & 119 & 6056.946 & 44.472 \\
\hline pb21 & 780 & 4200 & 94 & 158 & 6057.702 & 49.548 \\
\hline pb22 & 880 & 4750 & 91 & 140 & 4938.707 & 61.796 \\
\hline pb23 & 1221 & 7216 & 356 & 1495 & 5775.604 & 604.440 \\
\hline pb24 & 1474 & 8734 & 128 & 174 & 1586.150 & 408.272 \\
\hline pb25 & 1386 & 8206 & 84 & 130 & 2636.618 & 200.364 \\
\hline pb26 & 972 & 6186 & 109 & 143 & 2162.484 & 112.396 \\
\hline pb27 & 1356 & 8682 & 164 & 232 & 5961.284 & 437.956 \\
\hline pb28 & 864 & 5484 & 114 & 153 & 3022.051 & 82.972 \\
\hline pb29 & 2145 & 14859 & 208 & 311 & 4805.068 & 2137.156 \\
\hline pb30 & 1898 & 13130 & 137 & 200 & 2994.086 & 988.284 \\
\hline
\end{tabular}

Table 4: Numerical results obtained by applying the direct solution method only in the zone, and computing the PMP-based solution in the prezone as well as in the postzone: number of variables, number of constraints, number of iterations, number of funtion evaluations, objective value, computing time. 
Objective function

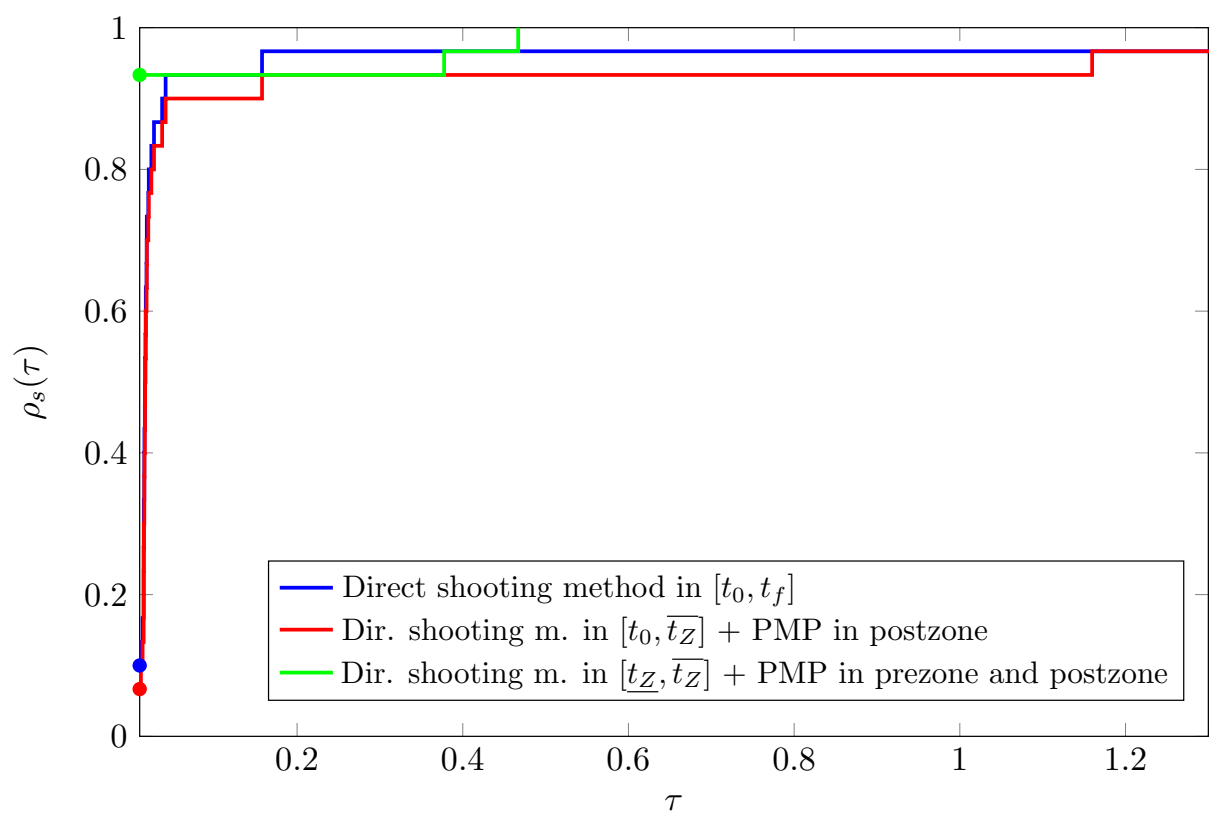

Figure 3: Performance profile on the value of the objective function. 


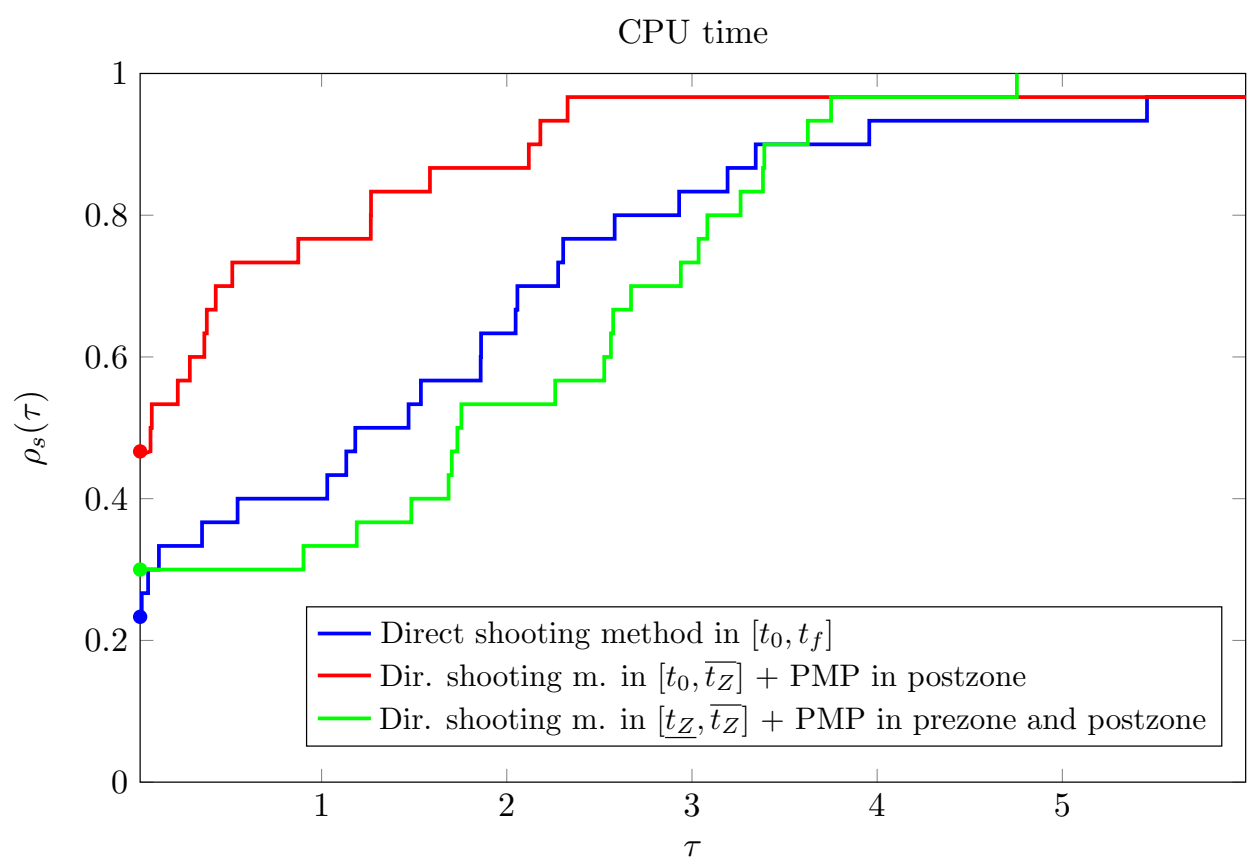

Figure 4: Performance profile on the computing time.

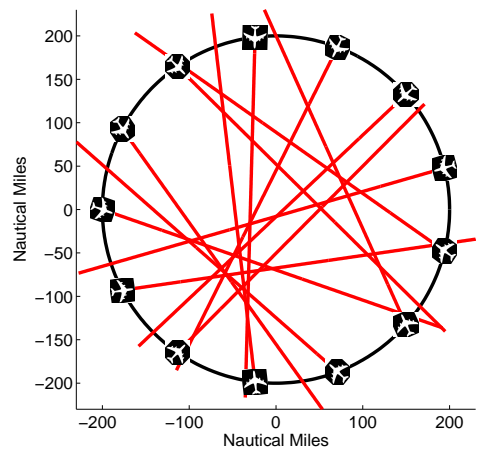

Figure 5: $\mathbb{R}^{2}$-trajectory configuration for the 13 -aircraft conflict avoidance problem pb29. 


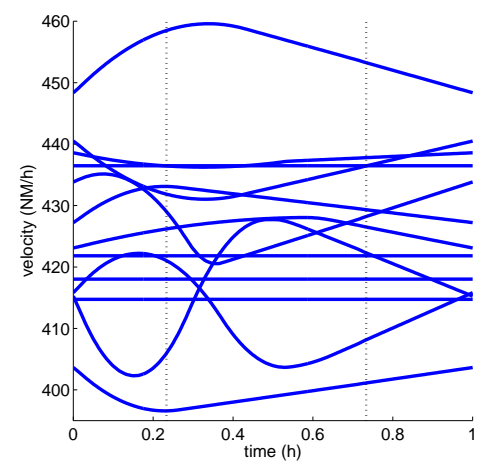

Figure 6: Optimal velocity solution curves for the 13-aircraft conflict avoidance problem pb29.

which are solved by using numerical direct shooting methods. Numerical tests on thirty problem instances show that formulation $(\mathcal{P}$ postzone $)$ provides the best results in terms of computing time, while formulation ( $\mathcal{P}$ prezone) provides a solution on all instances, that are also the most smooth solutions.

The main advantages of our approaches are the continuity of the velocity solutions, the subliminal velocity variations as suggested in the European ERASMUS project, the accuracy on the state informations (velocities and positions, along the whole time window), and the reasonable computing time (for small number of aircraft involved, e.g., less than 0.5 seconds for a problem involving 4 aircraft along a one-hour time horizon, using the formulation ( $\mathcal{P}$ prezone).

\section{Acknowledgment}

The authors gratefully acknowledge financial support by French National Research Agency (ANR) under grant ANR 12-JS02-009-01 "ATOMIC" (http://atomic.recherche.enac.fr/). 


\section{References}

[1] A. Alonso-Ayuso, L. F. Escudero, and F. J. Martín-Campo. A mixed 01 nonlinear optimization model and algorithmic approach for the collision avoidance in ATM: velocity changes through a time horizon. Computers $\mathcal{E}$ Operations Research, 39(12):3136-3146, 2012.

[2] A. Alonso-Ayuso, L. F. Escudero, and F. J. Martn-Campo. Exact and approximate solving of the aircraft collision resolution problem via turn changes. Transportation Science, 50(1):263-274, 2016.

[3] A. Bicchi and L. Pallottino. On optimal cooperative conflict resolution for air traffic management systems. IEEE Transactions on Intelligent Transportation Systems, 1(4):221-231, 2000.

[4] D. Bonini, C. Dupré, and G. Granger. How ERASMUS can support an increase in capacity in 2020. In Proceedings of CCCT: the $7^{\text {th }}$ International Conference on Computing, Communications and Control Technologies, 2009.

[5] J. Bonnans. The shooting approach to optimal control problems. In Proceedings of IFAC ALCOSP: the $11^{\text {th }}$ IFAC International Workshop on Adaptation and Learning in Control and Signal Processing, 2013.

[6] M. Brochard. ERASMUS - en route air traffic soft management ultimate system. Technical report, Eurocontrol Experimental Centre, 2006.

[7] R. Bulirsch, E. Nerz, H. Pesch, and O. von Stryk. Combining direct and indirect methods in optimal control: range maximization of a hang glider. ISNM International Series of Numerical Mathematics, 111:273-288, 1993.

[8] S. Cafieri. MINLP in Air Traffic Management: Aircraft conflict avoidance. In T. Terlaky, M. Anjos, and S. Ahmed, editors, Advances and Trends in Optimization with Engineering Applications, MOS-SIAM Series on Optimization, pages 293-301. SIAM, Philadelphia, 2017.

[9] S. Cafieri and N. Durand. Aircraft deconfliction with speed regulation: new models from mixed-integer optimization. Journal of Global Optimization, 58(4):613-629, 2014.

[10] S. Cafieri and R. Omheni. Mixed-integer nonlinear programming for aircraft conflict avoidance by sequentially applying velocity and heading angle changes. European Journal of Operational Research, 260(1):283-290, 2017. 
[11] L. Cellier. Evitement de conflits aériens par une régulation subliminale en vitesse : modélisation $\&$ résolution via le contrôle optimal. PhD thesis, Université Toulouse 3 Paul Sabatier, ENAC, Toulouse, France, 2015.

[12] L. Cellier, S. Cafieri, and F. Messine. Optimal control approaches for aircraft conflict avoidance using speed regulation: a numerical study. In Proceedings of the ISIATM 2013, Interdisciplinary Science for Innovative Air Traffic Management, Toulouse, France, 2013.

[13] J. C. Clements. Minimum-time turn trajectories to fly-to points. Optimal Control Applications and Methods, 11(1):39-50, 1990.

[14] J. C. Clements. The optimal control of collision avoidance trajectories in air traffic management. Transportation Research Part B, 33(4):265-280, 1999.

[15] E. Dolan and J. Moré. Benchmarking optimization software with performance profiles. Mathematical Programming Series A, 91(2):201-213, 2002.

[16] N. Durand. Optimisation de Trajectoires pour la Resolution de Conflits en Route. PhD thesis, Institut National Polytechnique de Toulouse, 1996.

[17] EEC. User manual for the base of aircraft data. Technical report, EEC Eurocontrol Experimental Centre, 2008.

[18] R. Fourer, D. Gay, and B. Kernighan. AMPL: a modeling language for mathematical programming. Thomson/Brooks/Cole, 2003.

[19] J. Kuchar and L. Yang. A review of conflict detection and resolution modeling methods. IEEE Transactions on Intelligent Transportation Systems, 1(4):179$189,2000$.

[20] L. Pallottino, E. M. Feron, and A. Bicchi. Conflict resolution problems for air traffic management systems solved with mixed integer programming. IEEE Transactions on Intelligent Transportation Systems, 3(1):3-11, 2002.

[21] C. Peyronne, A. R. Conn, M. Mongeau, and D. Delahaye. Solving air traffic conflict problems via local continuous optimization. European Journal of Operational Research, 241(2):502-512, 2015.

[22] L. Pontryagin, V. Boltyanski, R. Gamkrelidze, and E. Michtchenko. Théorie mathématique des processus optimaux. Editions Mir, Moscou, 1974.

[23] A. Richards and J. How. Aircraft trajectory planning with collision avoidance using mixed integer linear programming. In American Control Conference, 2002. Proceedings of the 2002, volume 3, pages 1936-1941 vol.3, 2002. 
[24] R. L. Schultz. Three-dimensional trajectory optimization for aircraft. Journal of Guidance, Control, and Dynamics, 13(6):936-943, 1990.

[25] A. Wächter and L. Biegler. On the implementation of primal-dual interior point filter line search algorithm for large-scale nonlinear programming. Mathematical Programming, 106(1):25-27, 2006. 